\title{
Stinging wasps, ants and bees (Hymenoptera: Aculeata) of the Murmansk region, Northwest Russia
}

\author{
Juho Paukkunen \& Mikhail V. Kozlov
}

Paukkunen, J. \& Kozlov, M. V. 2015: Stinging wasps, ants and bees (Hymenoptera: Aculeata) of the Murmansk region, Northwest Russia. — Entomol. Fennica 26: $53-73$.

\begin{abstract}
A total of 123 species of aculeate Hymenoptera are reported from the Murmansk region based on museum collections, unpublished datasets and previously published records. Collection localities are provided for each species. Erroneous or doubtful records of additional 19 species are discussed. Noteworthy findings include e.g. Ammophila campestris, Bombus consobrinus, B. hyperboreus, Crabro maeklini, Dipogon vechti, Formica suecica, Gorytes neglectus, Osmia cf. disjuncta and O. maritima. The species Ammophila campestris, Bombus veteranus, Crossocerus varus, Formica polyctena, Lindenius albilabris, Megachile analis and Osmia cf. disjuncta have their northernmost known localities in the Murmansk region. We estimate that approximately 100 additional aculeate species could still be found from the area.

J. Paukkunen, Finnish Museum of Natural History, Zoology unit, University of Helsinki, P.O. Box 17, FI-00014 Helsinki, Finland; E-mail: juho.paukkunen @helsinki.fi

M. V. Kozlov, Section of Ecology, University of Turku, FI-20014 Turku, Finland; E-mail:mikoz@utu.fi
\end{abstract}

Received 24 June 2014, accepted 21 November 2014

\section{Introduction}

The order Hymenoptera has been traditionally divided into three groups, according to the structure and life-style of the species: the sawflies (Symphyta), the parasitic wasps (Parasitica) and the aculeates (Aculeata). Of these groups only the aculeates, which include the stinging wasps, ants and bees, are considered to be monophyletic (Sharkey 2007). Usually the aculeates have been divided into three superfamilies, Chrysidoidea, Vespoidea and Apoidea (Aguiar et al. 2013), but recent molecular studies have shown that Vespoidea is paraphyletic with respect to Apoidea (Pilgrim et al. 2008).
The aculeates include many easily visible and economically significant taxa. The bees in particular play an essential role in the pollination of flowering plants, while social wasps and ants are important groups of predators. The females of most aculeates have a poisonous sting, which they use to defend themselves and to incapacitate their prey. Some species can sting humans and are therefore also of medical importance. Aculeates are warmth-loving insects, and the number of species decreases strongly towards the north in Europe. However, some species are restricted to the arctic region.

The Murmansk region (or oblast) of the Russian Federation is situated in northwestern Eu- 
rope and consists of the Kola Peninsula and some adjacent areas, most importantly Pechenga and Kuolajärvi (formerly Salla) in the west, and a small area south of Kandalaksha bay. The region is an integral part of Fennoscandia, as it has a common geological history with other areas of the physical-geographical area known as the Baltic shield. The insect fauna of the region is still poorly known compared to the rest of Fennoscandia, although detailed reviews have been published of Lepidoptera (Kozlov \& Jalava 1994, Kozlov \& Kullberg 2011). Several species of aculeate Hymenoptera have been reported in ecological and faunistic publications (e.g. Fridolin 1936, Uvarova \& Uvarov 1976, Polevoi \& Humala 2011). The only species list of all aculeates of the Murmansk region (Paukkunen \& Kozlov 2012) did not provide locality data and was published in Finnish in a low-circulation book, which limits its availability to the scientific community.

\section{History of the research}

The first preserved samples of aculeate Hymenoptera from the study area were probably collected by Johan (John) Reinhold Sahlberg in 1870. During a joint expedition with his fellow student, Aukusti Juhana Malmberg (later Mela), they collected at least 26 species of aculeates, as can be estimated from the materials deposited in the Finnish Museum of Natural History. Some of these records were published by Sahlberg (1889, 1910a, 1910b). A few species were collected later by Reinhold Enwald in 1880 (Enwald 1881), and by Klas Edgren in 1885 .

In 1887 , more than 200 specimens of about 35 species of aculeates were collected during the Great Kola Expedition, arranged by Finnish scientists (Rikkinen 1980). Collectors included Klas Edgren, Reinhold Enwald, Alfred Oswald Kihlman, Kaarlo Mainio Levander, Gustaf Nyberg and Johan Axel Palmén. Finnish biologists and entomologists, who collected aculeates from the Kola Peninsula prior to the Soviet revolution, included Bertil Poppius (expeditions in 1897 and 1899) and Justus Montell (one trip in 1899). Finally, Richard Frey and Wolter Hellen collected a large number of aculeate specimens of at least 36 species during their trip in 1913 (Frey 1915). For more details on collecting trips by Finnish entomologists, consult Silfverberg (1988).

No special research on the aculeate fauna of the Kola Peninsula was performed by Russian scientists in the pre-Soviet period. Although some material had been collected during 18941915 by at least ten people, the numbers of both specimens and species (deposited in the Zoological Museum in St. Petersburg) were relatively small. Collecting sites were mostly located near Kola fjord and Pechenga, on the northwestern coast of the peninsula.

As a consequence of the Tartu Peace of 1920, Finland obtained the Pechenga area (Petsamo) northwest of the Kola Peninsula, which became intensively studied during the 1920s and 1930s. During this period, about 1,200 specimens of aculeates belonging to 50 species were collected by nearly 30 entomologists, of which the most significant contributions were made by Wolter Hellén, Olavi Hulkkonen, Erkki Kanervo, Håkan Lindberg and Stephan Platonoff.

In the 1930s, insects of the Khibiny Mountains were studied by the ecologist Vladimir Yulievich Fridolin (Fridolin 1935, 1936). In addition to Fridolin, at least eight people collected aculeate material in the Kola Peninsula prior to the Second World War. This material consists of approximately 200 specimens of about 13 species. Collection localities, besides the Khibiny Mountains, included also the Kola Fjord and Yokanga River region, Svyatoi Nos peninsula and Kildin Island.

Between the Second World War and the breakup of the Soviet Union, collecting of aculeate Hymenoptera in the Kola Peninsula was very limited, although some scattered information was published by the Russian researchers (Striganova 1973, Semenov-Tyan-Shanskiy 1975, Uvarova \& Uvarov 1976, Byzova et al. 1986). The research intensified in the $2000 \mathrm{~s}$, as can be seen from publications on ants and other Hymenoptera of the Pasvik Nature Reserve (Makarova 2005, Mershchiev 2006, Polevoi \& Humala 2011), ants of the pollution-damaged forests of the Pechenga area (Mershchiev 2009), aculeates of the Khibiny region (Anufriev et al. 2011) and the pollinators of orchids (Blinova 2010, Blinova \& Mironov 2012). Also a largescale study on the environmental impacts of the 
Fig. 1. Borders of the Murmansk region and its subdivision into biogeographical provinces (modified after Urbanavichus et al. 2008). Kk: Karelia keretina, Ks: Regio kuusamoënsis, Li: Lapponia inarensis, Lim: Lapponia imandrae, Lm: Lapponia murmanica, Lp: Lapponia ponojensis, Lps: Lapponia petsamoënsis, $L t$ : Lapponia tulomensis and $L v$ : Lapponia varsugae.

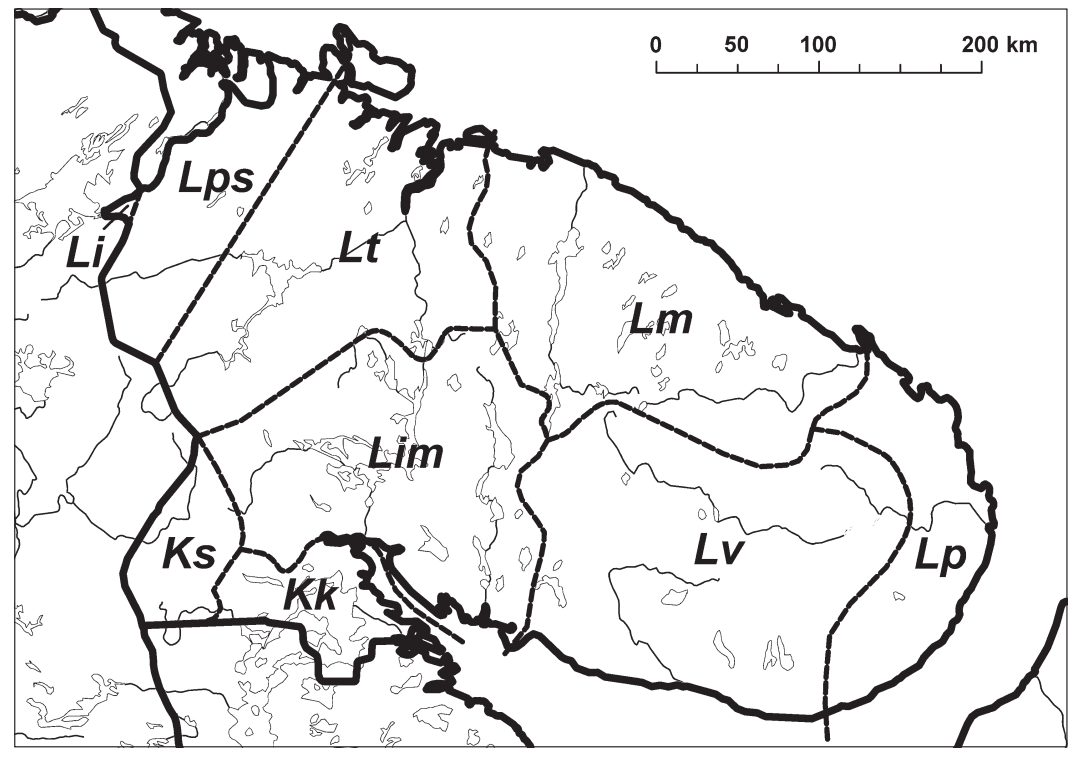

emissions of the Monchegorsk smelters, which has been continuing since the 1990s, has included the collection of large amounts of material on ants and bees (Kozlov 1997, Kozlov et al. 2009).

\section{Material and methods}

\subsection{Study area}

The Murmansk region has an area of $144,900 \mathrm{~km}^{2}$ and forms a part of the greater Lapland region that spans over four countries. Historically, it has also been referred to as Russian Lapland (Lapponia rossica, $L r$ ). The region lies almost completely north of the Arctic Circle and includes the entire Kola Peninsula. The area is generally hilly, with the Khibiny Mountains (altitude 1,201 m) forming the highest point of the region. The climate is mild due to the proximity of the sea and the Gulf Stream. The typical vegetation is northern taiga forest, dominated by Norway spruce (Picea abies (L.) Karst.) and Scots pine (Pinus sylvestris L.), which are replaced by mountain birch (Betula pubescens ssp. czerepanovii (N. I. Orlova) Hämet-Ahti) near the northern tree limit. The northernmost parts of the Kola Peninsula are covered by tundra. The study area includes nine biogeographical provinces, six of which are located entirely in the Murmansk region (Fig. 1).

\subsection{Collections}

The study is largely based on the collections of the Finnish Museum of Natural History, University of Helsinki (MZH, 1483 specimens of 107 species), the Zoological Museum of the University of Turku (ZMTu, 624 specimens of 18 species) and the Zoological Institute of the Russian Academy of Sciences in St. Petersburg (ZISP, approximately 950 specimens of 28 species, excluding ants which were not studied). The collections of MZH and ZMTu were studied and revised by JP, and information from the collections of ZISP was collected by MK. The identifications of most specimens in ZISP were not controlled during the recording of the label data, so their reliability was assessed subsequently based on the commonness and ease of identification of the species.

In addition to the museum collections, we used three unpublished ecological data sets collected by MK and his co-workers. The first set of samples was collected with yellow colour traps (i.e. so-called Russell traps) from 12 sites (located $1-47 \mathrm{~km}$ from Monchegorsk) in 2000 and comprised 2,282 aculeates of 17 species, identified by Guy Söderman. The second set, collected using the same methods from ten sites in 2010, was identified by JP and contained 394 individuals of 11 species. Bumblebees comprised approxi- 
Table 1. Localities from within or adjacent to which aculeates have been collected in the Murmansk region. Coordinates are given in the wgs84 system (northern latitude, eastern longitude) and error radiuses in kilometers. Number of recorded species is also presented for each locality.

\begin{tabular}{|c|c|c|c|c|}
\hline Locality & Province & Coordinates & Radius & Species \\
\hline Abram-Mys cape & $L t$ & $68.978,33.026$ & 0.5 & 1 \\
\hline Ainovy islands (= Heinäsaaret) & Lps & $69.838,31.571$ & 5 & 2 \\
\hline Ajkuajvenjok river & Lim & $67.565,33.774$ & 3 & 1 \\
\hline Alakurtti village (= Kurtti) & Ks & $66.960,30.340$ & 5 & 4 \\
\hline Alluaiv mountain & Lim & $67.873,34.445$ & 2 & 5 \\
\hline Apatity town & Lim & $67.566,33.391$ & 3 & 26 \\
\hline Berezovka village & Lim & $67.430,34.280$ & 0.5 & 4 \\
\hline Bolshaya Bogomolikha island & Kk & $67.072,32.509$ & 0.5 & 4 \\
\hline Bolshoe Goltsovoe lake (= Paikunjavr) & Lim & $67.852,33.670$ & 2 & 2 \\
\hline Bolshoi Lomnishnyi island & Kk & $66.970,32.626$ & 0.5 & 12 \\
\hline Bolshoi Vudjavr lake & Lim & $67.633,33.674$ & 2 & 1 \\
\hline Borisoglebskiy village (= Kolttaköngäs, Boris Gleb) & Lps & $69.654,30.134$ & 1 & 10 \\
\hline Chalmozero lake & Lim & $67.655,31.487$ & 5 & 1 \\
\hline Chapoma village & $L p$ & $66.103,38.870$ & 0.5 & 5 \\
\hline Chavanga village & Lv & $66.109,37.756$ & 0.5 & 4 \\
\hline Chervyanaya village (= Kervanto) & Lps & $69.893,31.952$ & 0.5 & 3 \\
\hline Chuna village (Lapland Nature Reserve) & Lim & $67.661,32.647$ & 0.5 & 2 \\
\hline Chuna-tundra fells & Lim & $67.803,32.438$ & 15 & 1 \\
\hline Dalnie Zelentsy village & $L m$ & $69.117,36.062$ & 0.5 & 2 \\
\hline Devichya Luda island & $K k$ & $66.988,32.609$ & 0.5 & 8 \\
\hline Devyatoy cape & $L p$ & $66.880,41.244$ & 1 & 1 \\
\hline Dokucheikha island & Kk & $66.963,32.646$ & 0.5 & 4 \\
\hline Dolgaya Shchel fjord (= Peuravuono, Peuravuoma) & Lps & $69.723,31.209$ & 3 & 9 \\
\hline Ekaterininskiy island & $L t$ & $69.214,33.464$ & 1.5 & 2 \\
\hline Ekostrovski village (= Jokostrov) & Lim & $67.606,33.047$ & 10 & 7 \\
\hline Elmarajok river & Lim & $67.830,34.718$ & 2 & 9 \\
\hline Gagarka village (= Kaakkuri) & Lps & $69.550,31.247$ & 0.5 & 4 \\
\hline Gavrilovo village & $L m$ & $69.181,35.847$ & 0.5 & 8 \\
\hline Hakokoski rapids (Paz river) & Lps & $69.372,29.693$ & 0.5 & 3 \\
\hline Harjuvaara hill (= Harju) & Lps & $69.448,30.124$ & 1.5 & 3 \\
\hline Haukilampi lake & Lps & $69.439,30.768$ & 0.5 & 12 \\
\hline Imandra lake & Lim & $67.644,33.067$ & 50 & 8 \\
\hline Jäniskoski village & $\mathrm{Li}$ & $68.969,28.779$ & 0.5 & 2 \\
\hline Kalkupää fell & Lps & $69.293,29.331$ & 0.5 & 7 \\
\hline Kandalaksha town (= Kantalahti) & Lim & $67.156,32.402$ & 3 & 29 \\
\hline Kanozero lake & Lim & $67.036,34.127$ & 12 & 8 \\
\hline Kapustnoe village & Lim & $67.335,34.185$ & 0.5 & 5 \\
\hline Karablekk fell (= Korablekk) & Lps & $69.239,29.510$ & 0.5 & 1 \\
\hline Kashkarantsy village & Lv & $66.336,36.014$ & 0.5 & 13 \\
\hline Kaskama fell & Lps & $69.279,29.478$ & 1 & 7 \\
\hline Kaulatunturi fell & Lps & $69.404,30.246$ & 1 & 2 \\
\hline Khibiny mountains (= Umptek, Hiipinä) & Lim & $67.719,33.657$ & 25 & 5 \\
\hline Khibiny railway station & Lim & $67.673,33.211$ & 0.5 & 8 \\
\hline Kiddjaur lake (= Pitkäjärvi) & Lps & $69.450,31.185$ & 3 & 1 \\
\hline Kildin island (= Kiltinänsaari) & $L t$ & $69.349,34.178$ & 7 & 2 \\
\hline Kirovsk botanical garden & Lim & $67.648,33.670$ & 0.5 & 10 \\
\hline Kirovsk town & Lim & $67.615,33.663$ & 2 & 4 \\
\hline Kola fjord & $L t$ & $69.065,33.113$ & 20 & 2 \\
\hline Kola river & $L t$ & $68.767,33.149$ & 15 & 3 \\
\hline Kola town (= Kuola) & $L t$ & $68.881,33.020$ & 1.5 & 5 \\
\hline Köngäs rapids (Lotta river) & Lps & $68.576,29.408$ & 0.5 & 13 \\
\hline Korabelnoe village & $L p$ & $67.000,41.279$ & 0.5 & 2 \\
\hline Kuivakoski rapids (Lotta river) & Lps & $68.501,28.733$ & 0.5 & 2 \\
\hline
\end{tabular}




\begin{tabular}{|c|c|c|c|c|}
\hline Locality & Province & Coordinates & Radius & Species \\
\hline Kukisvumchorr mountain & Lim & $67.666,33.691$ & 1 & 2 \\
\hline Kunijok river & Lim & $67.813,33.615$ & 5 & 10 \\
\hline Kuolajärvi village (= old Salla) & Ks & $66.968,29.208$ & 0.5 & 41 \\
\hline Kuosmoaivi fell & Lps & $69.458,31.500$ & 2 & 4 \\
\hline Kurichek island & Kk & $66.993,32.580$ & 0.5 & 5 \\
\hline Kutsa river & Ks & $66.711,30.060$ & 15 & 2 \\
\hline Kuvernöörinkoski rapids (Kuvernöörinjoki river) & Lps & $69.512,30.464$ & 0.5 & 10 \\
\hline Kuzomen village & Lv & $66.287,36.865$ & 0.5 & 16 \\
\hline Kuzreka village & $L v$ & $66.605,34.811$ & 0.5 & 7 \\
\hline Lambin island & Kk & $67.065,32.461$ & 0.5 & 4 \\
\hline Laukkujoki river & Lps & $69.289,29.584$ & 2 & 2 \\
\hline Liinahamari village & Lps & $69.642,31.358$ & 0.5 & 2 \\
\hline Longvattn bay & Lps & $69.364,29.726$ & 1 & 3 \\
\hline Loparskaya valley & Lim & $67.681,33.798$ & 1 & 7 \\
\hline Lotta river (= Lutto) & Lps & $68.576,29.408$ & 40 & 15 \\
\hline Loustari village (= Yläluostari) & Lps & $69.426,31.057$ & 0.5 & 24 \\
\hline Lovozero mountains (= Lujaururt) & Lim & $67.825,34.690$ & 20 & 5 \\
\hline Maitokoski rapids (Paz river) & Lps & $69.402,29.791$ & 0.5 & 1 \\
\hline Malaya Belaya river (= Lutamarjok) & Lim & $67.680,33.291$ & 5 & 14 \\
\hline Maloe Goltsovoe lake & Lim & $67.838,33.672$ & 0.5 & 9 \\
\hline Malyi Lomnishnyi island & Kk & $66.971,32.607$ & 0.5 & 5 \\
\hline Malyi Vudjavr lake & Lim & $67.668,33.617$ & 1 & 41 \\
\hline Marjok river (= lower Afanasiya) & Lm & $67.781,35.344$ & 10 & 2 \\
\hline Mattert fell (= Matert) & Lps & $69.278,30.877$ & 1 & 4 \\
\hline Menikkajoki river & Lps & $69.390,29.905$ & 2 & 7 \\
\hline Monchegorsk town & Lim & $67.939,32.910$ & 3 & 44 \\
\hline Moncheguba bay & Lim & $67.922,33.053$ & 5 & 4 \\
\hline Nautsi village & Lps & $68.992,29.031$ & 1 & 19 \\
\hline Nikel town (= Kolosjoki) & Lps & $69.406,30.210$ & 1.5 & 15 \\
\hline Njuemmellampjok river (= Njemlomjok, upper Afanasiya) & a) $L v$ & $67.725,35.578$ & 10 & 5 \\
\hline Nota river (= Nuortijoki) & $L t$ & $68.115,29.908$ & 50 & 2 \\
\hline Notozero lake/village (= Nuortijärvi) & $L t$ & $68.558,31.223$ & 20 & 7 \\
\hline Olenegorsk town & Lim & $68.139,33.274$ & 2 & 2 \\
\hline Olenitsa village & Lv & $66.468,35.334$ & 0.5 & 11 \\
\hline Olenya Guba village & Lt & $69.213,33.373$ & 0.5 & 2 \\
\hline Ora village & Lt & $69.290,32.800$ & 0.5 & 1 \\
\hline Orshoaivi fell & Lps & $69.466,30.331$ & 2 & 10 \\
\hline Pak river & $L t$ & $68.773,32.360$ & 2 & 6 \\
\hline Pala Guba bay & $L t$ & $69.194,33.391$ & 1 & 1 \\
\hline Parkkina village (= Parkkino, Barkino) & Lps & $69.555,31.224$ & 1 & 19 \\
\hline Pasvik Nature Reserve & Lps & $69.141,29.242$ & 50 & 12 \\
\hline Paz river (= Paatsjoki, Pasvikelva) & Lps & $69.141,29.242$ & 50 & 2 \\
\hline Pechenga village / municipality (= Petsamo) & Lps & $69.555,31.224$ & 1 & 18 \\
\hline Pechengskie tundry fells (= Petsamontunturit) & Lps & $69.374,30.457$ & 15 & 10 \\
\hline Pitkäjärvi village & Lps & $69.297,29.564$ & 1 & 10 \\
\hline Pitkäloukko bay & Lps & $69.483,30.281$ & 2 & 2 \\
\hline Poachjok river & Lim & $67.682,33.603$ & 2 & 1 \\
\hline Polyarnyy town (= Alexandrovsk) & Lt & $69.203,33.455$ & 1.5 & 12 \\
\hline Ponoy river & $L v$ & $67.059,38.605$ & 120 & 4 \\
\hline Ponoy village & $L p$ & $67.076,41.126$ & 0.5 & 12 \\
\hline Porchinikha village & $L m$ & $69.079,36.248$ & 0.5 & 3 \\
\hline Port-Vladimir village (= Jeretik) & $L t$ & $69.415,33.113$ & 0.5 & 1 \\
\hline Pulonga river & $L p$ & $66.491,39.733$ & 30 & 1 \\
\hline Pulozero village & $L t$ & $68.358,33.298$ & 0.5 & 1 \\
\hline Pyalitsa village & $L p$ & $66.191,39.525$ & 0.5 & 1 \\
\hline Pyhäkuru gorge & Ks & $66.781,29.967$ & 1 & 10 \\
\hline Rajakoski village & Lps & $69.023,29.005$ & 0.5 & 1 \\
\hline
\end{tabular}




\begin{tabular}{|c|c|c|c|c|}
\hline Locality & Province & Coordinates & Radius & Species \\
\hline Revda town & Lim & $67.942,34.555$ & 1 & 3 \\
\hline Ryazhkov island & $K k$ & $67.017,32.557$ & 2 & 16 \\
\hline Saarikoski rapids (Paz river) & Lps & $69.528,30.146$ & 0.5 & 1 \\
\hline Sairaalantunturi fell & Lps & $69.561,31.217$ & 0.5 & 4 \\
\hline Sallatunturi fell & Ks & $66.906,29.230$ & 1 & 1 \\
\hline Salmijärvi village & Lps & $69.437,30.125$ & 3 & 16 \\
\hline Seidozero lake (= Seitjaur) & Lim & $67.817,34.850$ & 5 & 4 \\
\hline Solovaraka hill (= Solovareka) & $L t$ & $68.873,33.021$ & 0.5 & 7 \\
\hline Spasitelnaya Gora fell (= Pelastusvuori) & Lps & $69.417,31.094$ & 0.5 & 1 \\
\hline Srednyaya Guba bay & $L t$ & $69.141,33.572$ & 1.5 & 3 \\
\hline Svetloe lake (= Vavnjarg) & Lim & $67.912,34.956$ & 1 & 1 \\
\hline Svyatoy Nos cape & $L p$ & $68.096,39.852$ & 8 & 2 \\
\hline Tetrino village & Lv & $66.064,38.246$ & 0.5 & 3 \\
\hline Tri Ostrova village & $L p$ & $67.210,41.318$ & 0.5 & 1 \\
\hline Trifonajärvi lake & Lps & $69.628,31.249$ & 3 & 5 \\
\hline Trifonovo village (= Trifona) & Lps & $69.595,31.266$ & 0.5 & 9 \\
\hline Tuljok river & Lim & $67.719,33.827$ & 4 & 6 \\
\hline Tuloma river (= Tuulomajoki) & $L t$ & $68.765,32.410$ & 40 & 2 \\
\hline Tumannyy village & $L m$ & $68.882,35.655$ & 0.5 & 6 \\
\hline Töllevi village & Lps & $69.541,30.207$ & 1 & 2 \\
\hline Umba river & Lim & $67.171,34.071$ & 50 & 2 \\
\hline Umba village & Lim & $66.697,34.347$ & 3 & 11 \\
\hline Umbozero lake (= Umpjaur) & Lim & $67.714,34.385$ & 30 & 1 \\
\hline Vaarlam island (= Vaarlamansaari, Niilansaari) & Lps & $69.141,29.247$ & 1 & 16 \\
\hline Varzino village & $\mathrm{Lm}$ & $68.359,38.391$ & 1 & 1 \\
\hline Varzuga village & Lv & $66.393,36.586$ & 2 & 5 \\
\hline Vavnbed mountain & Lim & $67.920,35.004$ & 2 & 3 \\
\hline Vayda-Guba village (= Vaitolahti) & Lps & $69.936,31.970$ & 1 & 5 \\
\hline Verkhnetulomskiy village (= Ylä-Tuuloma) & $L t$ & $68.605,31.792$ & 1 & 11 \\
\hline Verkhniy Nyud village & Lim & $67.863,32.932$ & 0.5 & 5 \\
\hline Virma river (= Varmjok) & $L m$ & $68.033,34.921$ & 10 & 2 \\
\hline Viteguba bay & Lim & $67.801,32.902$ & 5 & 2 \\
\hline Voronya river & $L m$ & $68.633,35.654$ & 60 & 4 \\
\hline Voronye village (= Voroninsk) & $L m$ & $68.455,35.344$ & 10 & 6 \\
\hline Voryema village (= Vuoremi) & Lps & $69.786,30.827$ & 0.5 & 9 \\
\hline Vostochnaya Litsa village (= Litsa) & $\mathrm{Lm}$ & $68.643,37.800$ & 1 & 1 \\
\hline Vudjavrchorr mountain & Lim & $67.623,33.602$ & 1 & 1 \\
\hline Vudjavrjok river & Lim & $67.679,33.654$ & 5 & 11 \\
\hline Vuorijärvi village (= Vuorikylä) & Ks & $66.789,30.158$ & 1 & 16 \\
\hline Yokanga village (= Jokanga, lokanga) & $L m$ & $68.001,39.701$ & 0.5 & 4 \\
\hline Zapolyarnyy town & Lps & $69.426,30.820$ & 1 & 3 \\
\hline Zasheek village & Lim & $67.407,32.558$ & 2 & 3 \\
\hline Zemlyanoe village (= Pummanki) & Lps & $69.786,31.961$ & 0.5 & 13 \\
\hline
\end{tabular}

mately $97 \%$ of the individuals and $75 \%$ of the species in both samples. The third set of samples collected with pitfall traps from ten sites in 2009 was identified by Dmitry A. Dubovikoff and included 1,048 ants belonging to 13 species.

\subsection{Publications}

We carefully searched all published data on aculeates from the Kola Peninsula, including pa- pers written in Finnish and Russian. All records were examined for the reliability of identifications by JP. In addition to the literature mentioned in the introduction, records of aculeates were extracted from the following publications: Anufriev et al. (2011), Collingwood (1979), Elfving (1968), Forsius and Nordström (1921, 1923), Friese (1911), Grönblom (1916), Hellén (1920a, 1920b, 1953, 1954, 1955), Lomholdt (1975, 1976), Løken (1973), Nordström and Forsius 
(1928), Olmi (1994), Pekkarinen and Huldén (1991, 1995), Pekkarinen et al. (1981), Pulkkinen (1931), Shutova (2003), Skorikov (1912, 1914), Söderman and Leinonen (2003), Wolf(1967) and Zvereva et al. (2010).

\subsection{Nomenclature and data presentation}

The nomenclature mainly follows the checklist of Finnish aculeates (Paukkunen 2010a). Families and species within the families are listed in alphabetical order. For rare species with not more than five localities we include full data; for common species we only list the collecting localities in alphabetical order (for the coordinates, consult Table 1) within each biogeographical province (also in alphabetical order). The province abbreviation is underlined, if the observation is based on a specimen identified or verified by Juho Paukkunen, Pekka Punttila (Formicidae), Jouni Sorvari (Formicidae) or Veli Vikberg (Dryinidae). References to published data are given for rare species but omitted for common species. Noteworthy species are provided with brief comments on their distribution.

Abbreviations: is. $=$ island, islands; $\mathrm{r} .=$ river; $1 .=$ lake; st. = railway station; $\mathrm{mt} .=$ mountain; $\mathrm{mts} .=$ mountains; low. $=$ lower course; mid. $=$ middle course; upp. = upper course; sur. = surroundings; W. = west; E. = east; S. = south; N. = north .

\section{Species found from the Murmansk region}

\subsection{Family Andrenidae}

Andrena barbilabris (Kirby, 1802)

Lm: $12 \mathrm{~km} \mathrm{~W}$. of Tumannyy near Voronya river 12.VII.2006 19 (Lvovsky).

Andrena clarkella (Kirby, 1802)

Lim: Monchegorsk sur. Lps: Kalkupää; Laukkujoki r.; Loustari; Nikel; Vaarlam is.; Zapolyarnyy sur. $L v$ : Kuzomen.

Andrena haemorrhoa (Fabricius, 1781)

Lim: 1 km E. of Apatity 29.VI.2012 1ㅇ (Blinova \& Mironov 2012: 80).
Andrena lapponica Zetterstedt, 1838

Ks: Alakurtti; Kuolajärvi. Lim: Bolshoi Vudjavr 1.; Kandalaksha; Kirovsk; Malaya Belaya r.; Malyi Vudjavr 1.; Monchegorsk sur. (10 localities). Lm: Voronye. Lps: Gagarka; Hakokoski; Haukilampi 1.; Kaskama; Lotta r.; Loustari; Nautsi; Nikel; Orshoaivi; Parkkina; Pitkäjärvi; Trifonajärvi 1.; Voryema. Lt: Kola town; Solovaraka; Tuloma r. $\underline{L v}$ : Kuzomen; Kuzreka.

Andrena ruficrus Nylander, 1848

Lim: 9 km S. of Monchegorsk 18.VI.2003 1 우 (Kozlov); Malyi Vudjavr 1. (Fridolin 1936). Lv: Kuzomen 17.VI.1913 1ð̊ (Frey).

\subsection{Family Apidae}

Apis mellifera Linnaeus, 1758

Lv: Varzuga 6.VIII.2011 1 worker (Paukkunen). The honey bee does not survive in the area without the help of beekeepers. According to Semenov-Tyan-Shanskiy (1975), the honey bee was introduced to the Murmansk region in 1949 for the pollination of cucumbers in greenhouses.

Bombus alpinus (Linnaeus, 1758)

Lim: Alluaiv mt.; Imandra 1.; Kirovsk; Kirovsk botanical garden; Loparskaya valley; Maloe Goltsovoe 1.; Malyi Vudjavr 1.; Tuljok r. Lm: Gavrilovo; Porchinikha; Tumannyy sur.; Yokanga. Lp: Korabelnoe sur.; Ponoy; Svyatoy Nos. Lps: Borisoglebskiy 1.; Dolgaya Shchel; Haukilampi 1.; Parkkina; Pechenga; Pechengskie tundry; Trifonajärvi 1.; Vayda-Guba; Voryema; Zemlyanoe. Lt: Olenya Guba; Pala Guba; Polyarnyy; Srednyaya guba. The taxon Bombus alpinus var. diabolicus, described by Friese (1911) from the Kola Peninsula, is currently synonymized with $B$. polaris.

Bombus balteatus Dahlbom, 1832

Ks: Kuolajärvi. Lim: Imandra 1.; Kandalaksha; Kukisvumchorr mt.; Lovozero mts.; Malyi Vudjavr 1.; Monchegorsk sur. (6 localities). Lm: Gavrilovo; Tumannyy sur. Lps: Ainovy is.; Borisoglebskiy; Dolgaya Shchel; Haukilampi 1.; Kaulatunturi; Kuosmoaivi; Kuvernöörinkoski; Loustari; Orshoaivi; Parkkina; Pechenga; Pechengskie tundry; Salmijärvi; Trifonajärvi 1.; Trifonovo; Zemlyanoe. Lt: Ekaterininskiy is.; 
Murmansk; Notozero 1.; Polyarnyy. $\underline{L v}$ : Njuemmellampjok r.

Bombus bohemicus Seidl, 1837

Lim: Elmarajok r. (Anufriev et al. 2011); $29 \mathrm{~km} \mathrm{S.}$ of Monchegorsk 20001 ex. (Kozlov); Umba 27.30.VI.1887 1ㅇ (Edgren). Lv: Kuzomen 16.22.VI.1913 2우 (Hellén \& Frey). "Lapponia rossica" (Sahlberg 1889: 171, as Apathus vestalis).

Bombus cingulatus Wahlberg, 1855

$\underline{K S}$ : Kuolajärvi; Pyhäkuru. Lim: Imandra 1.; Kandalaksha; Malyi Vudjavr 1.; Monchegorsk sur.; Moncheguba; Verkhniy Nyud. Lps: Kaskama; Parkkina. Lt: Verkhnetulomskiy.

Bombus consobrinus Dahlbom, 1832

Lp: Ponoy 15.VII.1913 1ㅇ (Hellén). $\underline{L v}$ : Kuzomen 16.VI.1913 1q (Hellén). "Lapponia rossica" (Sahlberg 1889: 171). "Kola Peninsula" (Skorikov 1914: 286, as Hortobombus consobrinus ssp. sahlbergi). The closest populations of this species outside the Kola Peninsula are in Abisko, northern Sweden, and on the northwest coast of Lake Onega in Russian Karelia. In Finland, the species is very rare and has been classified as critically endangered (Paukkunen 2010b). Females collect pollen only from the flowers of Aconitum lycoctonum L., Ranunculaceae (Løken 1973).

Bombus distinguendus Morawitz, 1869 Lim: Apatity 30.VI.1999 1 worker (Kozlov).

Bombus flavidus Eversmann, 1852

$\underline{K}$ : Sallatunturi. Lim: Kirovsk; Malyi Vudjavr 1.; Monchegorsk sur. (5 localities). Lps: Borisoglebskiy; Hakokoski; Kuvernöörinkoski; Loustari; Parkkina; Pechenga; Pechengskie tundry; Salmijärvi; Zemlyanoe. Lt: Notozero 1. Lv: Ponoy r. mid.

Bombus hypnorum (Linnaeus, 1758)

$K k$ : Ryazhkov is. Ks: Kuolajärvi. Lim: Apatity sur.; Elmarajok r.; Kirovsk botanical garden; Malaya Belaya r.; Maloe Goltsovoe 1.; Malyi Vudjavr 1.; Monchegorsk sur. (12 localities); Tuljok r. Lps: Kuvernöörinkoski; Loustari; Menikkajoki r.; Nautsi; Orshoaivi; Parkkina; Pechengskie tundry; Salmijärvi; Vaarlam is. $\underline{L t}$ : Kola; Polyarnyy.
Bombus hortorum (Linnaeus, 1761)

Lim: Apatity and its sur.; Elmorajok r. upp.; Kirovsk botanical garden; Monchegorsk sur.; Revda; Umba r. upp. Lps: Dolgaya Shchel; Trifonovo. Lt: Kola.

Bombus hyperboreus Schönherr, 1809

Lim: Malyi Vudjavr 1. 81 exx. (various collectors); Elmorajok r. upp. (Anufriev et al. 2011). Lm: Gavrilovo 27.VII.1913 2రิðَ (Hellén); Gavrilovo 23.VII.1913 1 worker (Frey); Varzino 5.VIII.1887 1 우 (Enwald). Lp: Svyatoy Nos 14.15.VIII.1880 1, 2 workers (Enwald) (Enwald 1881: 255, Sahlberg 1889: 171). Lps: Pechenga (Løken 1973: 116). In Fennoscandia this rare species is confined to the high alpine and tundra regions. It has been classified as near threatened in Finland (Paukkunen 2010b). The queens usually do not set up their own nests, but lay their eggs in the nests of $B$. polaris or $B$. alpinus, in which the larvae develop as inquilines (Söderman \& Leinonen 2003).

Bombus jonellus (Kirby, 1802)

$\underline{K k}$ : Ryazhkov is. $\underline{K s}$ : Kuolajärvi. Lim: Alluaiv mt.; Apatity sur.; Ekostrovski; Elmarajok r.; Kandalaksha; Kanozero 1.; Kapustnoe; Khibiny st.; Kirovsk botanical garden; Loparskaya valley; Lovozero mts.; Maloe Goltsovoe 1.; Malyi Vudjavr 1.; Monchegorsk and its sur. (15 localities); Moncheguba; Olenegorsk sur.; Tuljok r.; Umba; Vavnbed; Viteguba; Vudjavrjok r. Lm: Gavrilovo; Marjok r. low.; Tumannyy sur.; Voronya r.; Voronye; Yokanga. Lp: Ponoy. Lps: Ainovy is.; Borisoglebskiy; Chervyanaya; Dolgaya Shchel; Haukilampi 1.; Kalkupää; Kuosmoaivi; Kuvernöörinkoski; Lotta r.; Loustari; Mattert; Menikkajoki r.; Nautsi; Orshoaivi; Parkkina; Pechenga; Pechengskie tundry; Pitkäjärvi; Pitkäloukko; Salmijärvi; Trifonovo; Vayda-Guba; Vaarlam is.; Zemly-

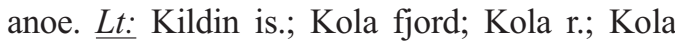
town; Murmansk; Notozero 1.; Pak r. low.; Polyarnyy; Solovaraka; Verkhnetulomskiy. $L v$ : Chavanga; Kashkarantsy; Kuzomen; Njuemmellampjok r.; Olenitsa; Ponoy r. mid.; Ponoy r. upp.; Tetrino; Varzuga.

Bombus lapponicus (Fabricius, 1793)

Ks: Kuolajärvi. Lim: Alluaiv mt.; Chalmozero 1.; Ekostrovski; Elmarajok r.; Imandra 1.; 
Kandalaksha; Kirovsk botanical garden; Kunijok r.; Loparskaya valley; Lovozero mts.; Malaya Belaya r.; Maloe Goltsovoe 1.; Malyi Vudjavr 1.; Monchegorsk sur. (10 localities); Moncheguba; Poachjok r.; Seidozero 1.; Tuljok r.; Vavnbed; Vudjavrjok r. Lm: Gavrilovo; Porchinikha; Tumannyy sur.; Voronya r.; Voronye; Yokanga. Lp: Chapoma; Korabelnoe sur.; Ponoy. Lps: Ainov is.; Borisoglebskiy; Dolgaya Shchel; Gagarka; Harjuvaara; Haukilampi 1.; Kalkupää; Kaskama; Kuosmoaivi; Kuvernöörinkoski; Liinahamari; Loustari; Mattert; Menikkajoki r.; Nautsi; Nikel; Orshoaivi; Parkkina; Pechenga; Pechengskie tundry; Pitkäjärvi; Salmijärvi; Trifonajärvi 1.; Trifonovo; Vayda-Guba; Vaarlam is.; Voryema; Zemlyanoe. Lt: Abram-Mys; Ekaterininskiy is.; Kildin is.; Kola fjord; Kola r.; Kola town; Olenya Guba; Port-Vladimir; Murmansk; Notozero 1.; Ora; Polyarnyy; Solovaraka; Srednyaya guba; Tuloma r. (at "Krivets-Padun"); Verkhnetulomskiy. Lv: Chavanga; Kuzomen; Tetrino. Skorikov (1912) described many new forms (subspecies, varieties and aberrations) of Bombus lapponicus from the Kola Peninsula based on slight differences in coat colouration. However, as the colour variation is considerable and continuous in B. lapponicus, these forms have little taxonomic value and should be considered as synonyms.

\section{Bombus lucorum group}

Ks: Kuolajärvi. Lim: Alluaiv mt.; Apatity sur.; Berezovka; Bolshoe Goltsovoe 1.; Ekostrovski; Elmarajok r.; Khibiny st.; Kirovsk botanical garden; Loparskaya valley; Malyi Vudjavr 1.; Monchegorsk sur. (13 localities); Revda; Umba; Verkhniy Nyud; Vudjavrjok r. Lm: Yokanga. Lps: Dolgaya Shchel; Kuvernöörinkoski; Nautsi; Loustari; Parkkina; Pechenga; Pechengskie tundry; Salmijärvi; Trifonovo; Zemlyanoe. Lt: Kola town; Pak r. low.; Polyarnyy; Verkhnetulomskiy. Lv: Chavanga; Kashkarantsy; Kuzomen; Kuzreka; Njuemmellampjok r.; Olenitsa; Ponoy r. mid.; Varzuga. We have not been able to discriminate among the cryptic species of the Bombus lucorum group, i.e. Bombus lucorum, $B$. cryptarum and B. magnus. In North Europe, these species are morphologically very similar, and reliable identification is possible only through DNA barcoding or by analyzing labial gland se- cretions of males (Bertsch 2009). It is possible that both B. lucorum and B. cryptarum occur in the Kola Peninsula.

\section{Bombus monticola Smith, 1849}

Lm: Gavrilovo. Lp: Ponoy. Lps: Dolgaya Shchel; Liinahamari; Loustari; Orshoaivi; Parkkina; Pechengskie tundry; Pitkäjärvi; Trifonajärvi 1.; Zemlyanoe. This species is difficult to distinguish morphologically from B. lapponicus (Gjershaug et al. 2013), and a more reliable assessment of its distribution on the Kola Peninsula would require the application of molecular methods.

Bombus pascuorum (Scopoli, 1763)

Ks: Kuolajärvi. Lim: Apatity sur.; Elmorajok r. upp.; Kandalaksha; Khibiny st.; Kirovsk botanical garden; Malaya Belaya r.; Malyi Vudjavr 1.; Monchegorsk sur. (6 localities); Olenegorsk sur.; Revda; Seidozero 1.; Tuljok r.; Umba; Umba r. upp.; Verkhniy Nyud; Viteguba; Vudjavrjok r. Lp: Ponoy. Lps: Menikkajoki r.; Vaarlam is. Lt: Pak r. low. $L v$ : Olenitsa sur.; Varzuga.

Bombus pratorum (Linnaeus, 1761)

Ks: Kuolajärvi. Lim: Apatity sur.; Elmarajok r.; Imandra 1.; Khibiny st.; Kirovsk botanical garden; Kunijok r.; Loparskaya valley; Maloe Goltsovoe 1.; Malyi Vudjavr 1.; Monchegorsk sur. (8 localities); Moncheguba; Seidozero 1.; Umba; Vudjavrjok r. Lps: Borisoglebskiy; Haukilampi 1.; Kuvernöörinkoski; Loustari; Mattert; Orshoaivi; Parkkina; Pechenga; Pechengskie tundry; Pitkäloukko; Saarikoski; Salmijärvi; Trifonovo; Vaarlam is.; Zemlyanoe. Lt: Srednyaya guba; Verkhnetulomskiy.

Bombus polaris Curtis, 1835

Lim: Khibiny mts.; Lovozero mts. Lm: Gavrilovo; Voronya r. Lps: Chervyanaya; Dolgaya Shchel; Haukilampi 1.; Kaulatunturi; Loustari; Orshoaivi; Parkkina; Pechenga; Pechengskie tundry; Vayda-Guba; Zemlyanoe.

\section{Bombus sporadicus Nylander, 1848}

Kk: Ryazhkov is. Lim: Apatity sur.; Ekostrovski; Elmorajok r. upp.; Kunijok r. upp.; Loparskaya valley; Maloe Goltsovoe 1.; Monchegorsk and its sur. (7 localities); Seidozero 1.; Tuljok r.; Verkhniy Nyud; Malyi Vudjavr 1. Lt: Pak r. low. This is the only hymenopteran species included in the Red Book of the Murmansk region, in which it 
has been classified as a rare species, found only in the meadows surrounding Kandalaksha (Shutova 2003). However, the species is common and occurs abundantly for example in the Khibiny Mountains (Anufriev et al. 2011) and near Monchegorsk, also in severely polluted areas.

Bombus sylvestris (Lepeletier, 1832)

Lim: $20 \mathrm{~km}$ S. of Monchegorsk $20001 \mathrm{ex}$, (Kozlov); Malyi Vudjavr 1. 52 exx. (Fridolin) (Fridolin 1936: Table 5, as Psithyrus silvestria); Kirovsk botanical garden 4.VIII.2011 approxi-

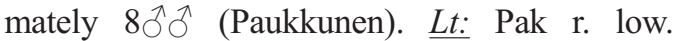
1.VIII.2011 3ठึ

Bombus veteranus (Fabricius, 1793)

Ks: Kuolajärvi 7.VIII.2011 1ठ (Paukkunen). Lim: Verkhniy Nyud 3.VIII.2011 1ð (Paukkunen).

Nomada leucophthalma (Kirby, 1802)

Lim: Malyi Vudjavr 1. (Fridolin 1936: 128).

Nomada panzeri Lepeletier, 1841

Ks: Kuolajärvi; Pyhäkuru. Lim: Ekostrovski; Kandalaksha; Kunijok r.; Malyi Vudjavr 1.; Monchegorsk sur. $\underline{L p}$ : Pulonga r. $\underline{\text { Lps: Gagarka; }}$ Lotta r.; Loustari; Nautsi; Nikel; Parkkina; Pitkäjärvi; Sairaalantunturi; Zapolyarnyy sur. Lt: 50 km E of Murmansk; Solovaraka.

\subsection{Family Bethylidae}

Bethylus berlandi Arlé, 1929

Lv: Kashkarantsy 20.-25.VII.1887 (Levander) (Hellén 1920b: 279 and 1953:91, as B. apteryx).

Bethylus fuscicornis (Jurine, 1807)

Lv: Kuzomen 22.VI.1913 2우 (Frey) (Hellén 1920b: 280, as B. fuscicornis f. syngenesiae).

\subsection{Family Chrysididae}

Elampus panzeri (Fabricius, 1804)

Ks: Kuolajärvi 16.VII.1938 1ð̄ (Y. Kangas).

Chrysis angustula Schenck, 1856

Ks: Kuolajärvi 26.VI.1938 1ठ (Y. Kangas).

Chrysis fulgida Linnaeus, 1761

Ks: Pyhäkuru 12.VII.1939 1ð (Lankiala). Lim: Malyi Vudjavr 1. 9.VIII.1937 1 우 (Fridolin).
Chrysis schencki Linsenmaier, 1968

$\underline{K s}$ : Kuolajärvi 1910s 1ठð (Nordström) (Hellén 1920a: 213, as C. ignita); Pyhäkuru 9.VII.1934 1 우 (Hellén); Vuorijärvi 11.VII.1934 1ㅇ (Hellén). Lps: Vaarlam is. (Humala \& Polevoi 2011: 278, as Chrysis sp.).

Chrysis sp. sensu Paukkunen et al. 2014

Lim: Kandalaksha 8.VII.1913 1ठ (Hellén). Lps: Loustari $1 \lesssim$ (Grönblom). Lt: $45 \mathrm{~km} \mathrm{E}$. of Murmansk 16.VII.2006 19 (Kozlov). Lv: Kashkarantsy 20.VII.1887 19 (Levander). This is an undescribed species, which is closely related to $C$. ignita and C. impressa (Paukkunen et al. 2014, Soon et al. 2014).

Chrysura hirsuta (Gerstaecker, 1869)

Lv: Chavanga 7.VIII.1887 1ㅇ (Levander) (Sahlberg 1910b: 97, as Chrysis osmiae; Hellén 1920a: 210, as Chrysis hirsuta).

\subsection{Family Colletidae}

Hylaeus annulatus (Linnaeus, 1758)

Ks: Kuolajärvi 8.-13.VII.1938 1ठ 1 우 (Y. Kangas), 29.VI.1937 1 ex. (E. Kangas), 20.VI.1938 19 (Karvonen). Lim: Kandalaksha 28.VI.1870 1ㅇ (Sahlberg); Malyi Vudjavr 1. (Fridolin 1936).

\subsection{Family Crabronidae}

Alysson ratzeburgi Dahlbom, 1843

Ks: Kuolajärvi; Vuorijärvi. Lim: Apatity sur.; Monchegorsk sur. Lps: Köngäs; Pechenga; Vaarlam is. $L v$ : Olenitsa.

Crabro lapponicus Zetterstedt, 1838

Lim: Kandalaksha 5.VII.1913 19 (Frey); Khibiny mts. 11.VII.1962 $10^{\hat{}}$ (Arens). $L v$ : Kuzreka 6.VII.1887 $1{ }^{\Uparrow}$ (Levander).

Crabro maeklini Morawitz, 1866

Lps: Haukilampi 1. 1ð (Kanervo); Loustari 9.VII.1930 1ð̋ (Hellén), 10.VII.1930 1q (Frey); Pechenga (Lomholdt 1976: 298). This rare species is known from Fennoscandia, Siberia, Mongolia, Ukraine and Kazakhstan (Pulawski 2011). It is classified as near threatened in Finland, Nor- 
way and Sweden (Paukkunen 2010b, Hansen et al. 2010, Cederberg et al. 2010).

Crossocerus barbipes (Dahlbom, 1845)

Ks: Kuolajärvi 24.IX.1938 1ð̊ (Y. Kangas).

Crossocerus dimidiatus (Fabricius, 1781)

Lim: $1 \mathrm{~km} \mathrm{~N}$. of Monchegorsk 28.VII.2005 1§

(Kozlov). Lps: Longvattn (Polevoi \& Humala 2011: 278).

Crossocerus leucostoma (Linnaeus, 1758)

Ks: Kuolajärvi. Lim: Kandalaksha; Khibiny mts.; Monchegorsk. Lps: Haukilampi 1.; Loustari; Salmijärvi. Lt: Kola.

Crossocerus nigritus Lepeletier \& Brullé, 1835

Lim: Malyi Vudjavr 1. 23.VII.1934 1 ( (Fridolin) (Fridolin 1936: 131, as Crabro pubescens).

Crossocerus ovalis Lepeletier \& Brullé, 1835

Lim: Apatity sur.; Kandalaksha; Kunijok r.; Malaya Belaya r.; Monchegorsk sur. Lps: Köngäs.

Crossocerus varus Lepeletier \& Brullé, 1835

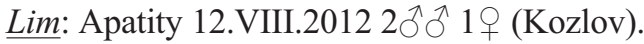

Crossocerus wesmaeli (Vander Linden, 1829)

Lim: $5 \mathrm{~km}$ N. of Monchegorsk 15.VII.2005 $2 \widehat{\jmath} \widehat{\jmath}$ (Kozlov); Khibiny mts. 20.VII.1928 1 ex. (Stark); Khibiny st. 27.VIII.1928 1 + (Cheburova). Lps: 0,8 km E. of Zapolyarnyy 1.VII.2005 1ठ (Kozlov).

Ectemnius borealis (Zetterstedt, 1838)

$\underline{K s:}$ : Vuorijärvi sur. ("Outonjoki") 26.VI.1936, $1+$ (Frey); Vuorijärvi 9.VII.1934 19 (Hellén). Lim: Khibiny st. 27.VIII.1928 1 ex. (Cheburova).

Ectemnius continuus (Fabricius, 1804)

$\underline{K s}$ : Kuolajärvi; Pyhäkuru; Vuorijärvi. Lim: Malaya Belaya r. $\underline{L p s}$ : Loustari; Parkkina. $\underline{L v}$ : Kashkarantsy.

Ectemnius guttatus (Vander Linden, 1829)

Lim: Khibiny st. 4.-15.VIII.1928 2 우 (Cheburova).

Gorytes neglectus Handlirsch, 1895

Lim: Kandalaksha 7.VII.1913 2ふふ઼ (Hellén), 8.VII.1913 1ㅇ (Frey) (Grönblom 1916: 29, Pulkkinen 1931: 63, Hellén 1954: 127, as $G$. fallax, Hellén 1955: 67). Lps: Lotta r. (at "Rohvinan kenttä") 11.VII.1899 1q (Poppius)
(Grönblom 1916: 29, Pulkkinen 1931: 63, Hellén 1954: 127, as G. fallax, Hellén 1955: 67). "Lapponia rossica" (Lomholdt 1975: 199). This is a rare, northern species which lives in sandy open areas, e.g. along rivers and on dry meadows. It is classified as near threatened in Finland (Paukkunen 2010b). In addition to Finland and the Murmansk region, it is known from the northern part of the Republic of Karelia, Siberia (Ural, Altai, Ussuri), Korea and Sakhalin (Pulawski 2011).

Lindenius albilabris (Fabricius, 1793)

$\underline{K s:}$ Kuolajärvi 7.VIII.2011 1ㅇ (Paukkunen).

Mimesa equestris (Fabricius, 1804)

Ks: Kuolajärvi 16.VII.1938 1ठ (Y. Kangas).

Lim: Apatity (Anufriev et al. 2011).

Mimumesa dahlbomi (Wesmael, 1852)

Lim: 9 km S. of Monchegorsk 24.VII.2004 1ठ (Kozlov).

Passaloecus monilicornis Dahlbom, 1842

Ks: Kuolajärvi 4.VII.1938 1q (Y. Kangas). Lps:

Vaarlam is. (Polevoi \& Humala 2011: 278).

Pemphredon inornata Say, 1824

Lim: Khibiny mts. 20.VII.1925 1 ex. (Stark). Lps: Karablekk (Polevoi \& Humala 2011: 278); $1.2 \mathrm{~km}$ E. of Nikel 7.VII.2004 1ठ઼ (Kozlov).

Pemphredon lugens Dahlbom, 1842

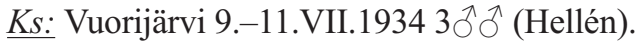

Pemphredon lugubris (Fabricius, 1793)

Lps: $\quad$ Haukilampi 1. 2.-8.VII.1937 19

(Nordman). Lv: Kashkarantsy 20.-25.VII.1887

$1{ }^{\hat{O}}$ (Edgren).

Pemphredon montana Dahlbom, 1845

Ks: Kuolajärvi 1910s 19 (Nordström); Kutsa r.

$2 ㅇ ㅜ$ (E. Kangas); Vuorijärvi 10.-11.7.1934 4 우 (Hellén).

Rhopalum clavipes (Linnaeus, 1758)

$\underline{K s}$ : Kuolajärvi 19 (Ritavuori). Lps: Vaarlam is. (Polevoi \& Humala 2011: 278).

Tachysphex pompiliformis (Panzer, 1805)

Lv: Kashkarantsy 20.VII.1887 1 (Levander).

Trypoxylon medium Beaumont, 1945

Lim: Imandra 1. 18.VI.1911 1ठํ (Solovyev). 


\subsection{Family Dryinidae}

Anteon pubicorne (Dalman, 1818)

Ks: Vuorijärvi 10.VII.1934 1ठ̋ (Hellén) (Hellén 1953: 99, as A. lucidus). "Lapponia rossica" (Olmi 1994: 56, 90).

Anteon subarcticum Hellén, 1953

Lps: Lotta r. near Köngäs 14.VII.1939 1 + (Platonoff) (Hellén 1953: 97).

Anteon tenuicorne (Dalman, 1823)

Ks: Alakurtti 8.VII.1934 1ठ (Hellén).

Gonatopus clavipes (Thunberg, 1827)

Lim: Kandalaksha 21.VII.1870 1 ㅇ (Sahlberg) (Sahlberg 1910: 14, as G. borealis; Hellén 1920b: 289, as G. pilosus; Hellén 1953: 94, as G. sepsoides). Lp: Devyatoy 5.-8.VIII.1870 1 ㅇ (Sahlberg) (Sahlberg 1910a: 9, as G. pilosus; Hellén 1920b: 289, as G. pilosus). "Lapponia rossica" (Olmi 1994: 83, 90).

Lonchodryinus daos (Walker, 1837)

Lp: Ponoy 17.VII.1913 1ठ (Frey).

Lonchodryinus longicornis (Dalman, 1823)

Lim: Kandalaksha 7.VII.1913 1ðَ (Frey) (Hellén 1920b: 285). Lp: Ponoy 13.-19.VII.1913 1 q (Hellén) (Hellén 1920b: 285). Lps: Salmijärvi 8.VII.1930 2 ふૈ (Hellén).

Lonchodryinus ruficornis (Dalman, 1818)

Ks: Vuorijärvi. Lim: Ekostrovski; between Imandra and Kandalaksha; Kandalaksha. Lp: Ponoy. Lps: Borisoglebskiy; Chervyanaya; Kalkupää (identification uncertain); Lotta r.; Loustari; Menikkajoki r. (identification uncertain); Vaarlam is. (identification uncertain). $\underline{\text { t: }}$ : Kola.

Lonchodryinus subapterus (Kieffer, 1905)

Lim: Ekostrovski 9.VII.1870 1ㅇ (Sahlberg) (Hellén 1920b: 285, as Anteon longicornis f. subaptera). "Lapponia rossica" (Hellén 1953: 100, as Prenanteon ruficornis f. subaptera).

\subsection{Family Formicidae}

Camponotus herculeanus (Linnaeus, 1758)

Kk: Bolshaya Bogomolikha is.; Bolshoi Lomnishnyi is.; Devichya Luda is.; Dokucheikha is.; Kurichek is.; Lambin is.; Malyi Lomnishnyi is.; Ryazhkov is. Ks: Kuolajärvi. Lim: Apatity sur.; Chuna-tundra; Elmarajok r.; Kandalaksha; Kanozero 1.; Kapustnoe; Kunijok r. upp.; Loparskaya valley; Lovozero mts.; Maloe Goltsovoe 1.; Malyi Vudjavr 1.; Monchegorsk sur. (11 localities). Lps: Nautsi; Lotta r.; Pasvik Nature Reserve. $L v$ : Kashkarantsy; Olenitsa sur.

Formica aquilonia Yarrow, 1955

$K k$ : Bolshoi Lomnishnyi is.; Ryazhkov is. $\underline{K S}$ : Kuolajärvi; Pyhäkuru. Lim: Kanozero 1.; Monchegorsk sur. (3 localities); Umba. Lps: Köngäs; Nautsi (identification uncertain); Pasvik Nature Reserve. Lv: Kuzomen sur.; Olenitsa sur.

Formica exsecta Nylander, 1846

$K k$ : Bolshoi Lomnishnyi is.; Ryazhkov is. $K s$ : Kuolajärvi. Lim: Malyi Vudjavr 1.; Monchegorsk sur. Lp: Chapoma. Lps: Köngäs; Nikel sur.; Pasvik Nature Reserve; Pechenga; Salmijärvi. $\underline{L v:}$ Njuemmellampjok r.; Olenitsa sur.

Formica gagatoides Ruzsky, 1904

$K k$ : Ryazhkov is. Ks: Kuolajärvi. Lim: Alluaiv mt.; Elmarajok r.; Kandalaksha; Kanozero 1.; Malaya Belaya r.; Malyi Vudjavr 1.; Monchegorsk and its sur.; Vudjavrjok r. $\underline{L m}$ : Dalnie Zelentsy; Virma r. $\underline{L p}$ : Chapoma. $\underline{L p s}$ : Borisoglebskiy; Harjuvaara; in between Salmijärvi and Loustari; Kaskama; Kuvernöörinkoski; Kuivakoski; Loustari; Maitokoski; Nautsi; Nikel and its sur.; Lotta r.; Orshoaivi; Parkkina; Pasvik Nature Reserve; Pechenga; Pitkäjärvi; Sairaalantunturi; Salmijärvi; Trifonovo; Töllevi; Voryema. Lt: Kola. Lv: Kuzomen; Kuzreka.

Formica lemani Bondroit, 1917

$K k$ : Bolshoi Lomnishnyi is.; Devichya Luda is.; Kurichek is.; Ryazhkov is. Ks: Kuolajärvi; Pyhäkuru. Lim: Apatity and its sur.; Kanozero 1.; Kapustnoe; Kunijok r.; Malaya Belaya r.; Monchegorsk sur. (11 localities); Vudjavrjok r. Lm: Voronye. Lp: Ponoy. Lps: Köngäs; Loustari; Nautsi (identification uncertain); Nikel and its sur.; Pechenga; Sairaalantunturi; Salmijärvi (identification uncertain). Lt: Kola; Solovaraka; Verkhnetulomskiy. $\underline{L v}$ : Kuzreka; Olenitsa.

Formica lugubris Zetterstedt, 1838

$K k$ : Bolshaya Bogomolikha is.; Bolshoi Lomnishnyi is.; Devichya Luda is.; Kurichek is.; Lambin is.; Ryazhkov is. Li: Jäniskoski. Lim: 
Elmorajok r. upp.; Kanozero 1.; Malaya Belaya r.; Maloe Goltsovoe 1.; Monchegorsk sur. Lps: Köngäs; Nikel and its sur.; Pasvik Nature Reserve; Pechenga. Lt: Kola town sur.; Solovaraka. Lv: Kashkarantsy sur.

Formica picea Nylander, 1846

Lim: Malyi Vudjavr 1. Lps: Nautsi; Nikel and its sur.; Pasvik Nature Reserve; Pechenga; Salmijärvi.

Formica polyctena Förster, 1850

Kk: Malyi Lomnishnyi is. (Byzova et al. 1986: 281). Li: Jäniskoski (Makarova 2005: 62). Lim: $35 \mathrm{~km} \mathrm{~S}$. of Monchegorsk (Zvereva et al. 2010: 614). Lv: Kuzomen 17.VI.1913 1 우 (Hellén); Kuzreka 8.-13.VII.1887 1 worker (Levander).

Formica sanguinea Latreille, 1798

Kk: Bolshoi Lomnishnyi is.; Devichya Luda is.; Ryazhkov is. Lim: Apatity sur.; Berezovka; Kandalaksha; Monchegorsk sur. (2 localities). Lps: Pasvik Nature Reserve.

Formica suecica Adlerz, 1902

Lv: Kuzomen 21.VI.1913 5 workers (Hellén \& Frey). The specimens were collected from sand dunes on the eastern side of the Varzuga river. The species is rare in Fennoscandia and has been classified as near threatened in Finland (Paukkunen 2010b).

Formica truncorum Fabricius, 1804

$K k$ : Bolshoi Lomnishnyi is.; Devichya Luda is.; Ryazhkov is. Ks: Kuolajärvi. Lim: Elmarajok r.; Monchegorsk sur. (5 localities). Lps: Nautsi; Nikel and its sur.; Pasvik Nature Reserve. $\underline{L t}$ : Verkhnetulomskiy sur.; Notozero 1. $\underline{L v}$ : Kashkarantsy.

Formica uralensis Ruzsky, 1895

Ks: Kuolajärvi 12.VII.1935 1 worker (Krogerus). Lps: Lotta r. N. of Köngäs 14.VII.1939 1 worker (Platonoff); Nautsi 1 worker (Kanervo); Parkkina Sairaalantunturi 2.VII.1929 1 worker (Håkan Lindberg); Pasvik Nature Reserve and its sur. (Mershchiev 2006: 164). "Lapponia rossica" (Collingwood 1979: 165).

Harpagoxenus sublaevis (Nylander, 1849)

Lim: $5 \mathrm{~km}$ S. of Monchegorsk 20091 ex. (Kozlov). Lps: Zemlyanoe 17.-20.VII.1930
2 우우 3 workers (Hellén). "Lapponia rossica" (Collingwood 1979: 161).

Leptothorax acervorum (Fabricius, 1793)

Kk: Bolshaya Bogomolikha is.; Bolshoi Lomnishnyi is.; Devichya Luda is.; Dokucheikha is.; Kurichek is.; Lambin is.; Malyi Lomnishnyi is.; Ryazhkov is. Ks: Kuolajärvi. Lim: Apatity sur.; Malyi Vudjavr 1.; Monchegorsk sur. (10 localities); Umba; Vudjavrjok r. Lm: Dalnie

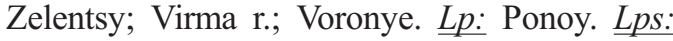
Borisoglebskiy; Dolgaya Shchel; Kuvernöörinkoski; Kuivakoski; Kuosmoaivi; Köngäs; Loustari; Nautsi; Nikel and its sur.; Paz r.; Parkkina; Pasvik Nature Reserve; Pechenga; Voryema; Zemlyanoe. Lt: Kola; Polyarnyy. $\underline{L v}$ : Kashkarantsy; Kuzomen; Olenitsa sur.

Myrmica lobicornis Nylander, 1846

$K k$ : Bolshoi Lomnishnyi is.; Devichya Luda is.; Dokucheikha is.; Malyi Lomnishnyi is.; Ryazhkov is. $\underline{K s}$ : Pyhäkuru. Lim: Malyi Vudjavr 1.; Monchegorsk sur. (4 localities); Umba.

Myrmica rubra (Linnaeus, 1758)

$K k$ : Bolshoi Lomnishnyi is.; Ryazhkov is. $\underline{K s}$ : Kuolajärvi. Lim: Apatity and its sur.; Malaya Belaya r.; Malyi Vudjavr 1.; Monchegorsk sur. (4 localities); Vudjavrjok r.

Myrmica ruginodis Nylander, 1846

$K k$ : Bolshaya Bogomolikha is.; Bolshoi Lomnishnyi is.; Dokucheikha is.; Kurichek is.; Lambin is.; Malyi Lomnishnyi is.; Ryazhkov is. Ks: Alakurtti; Kuolajärvi. Lim: Apatity sur.; Kanozero; Malaya Belaya r.; Malyi Vudjavr 1.; Monchegorsk sur. (11 localities); Umba; Vudjavrjok r. Lps: Borisoglebskiy; Harjuvaara; Kaskama; Nikel sur.; Pasvik Nature Reserve; Pechenga; Salmijärvi; Trifonovo. Lt: Kola.

Myrmica scabrinodis Nylander, 1846

$K k$ : Bolshoi Lomnishnyi is. and Ryazhkov is. (Byzova et al. 1986: 281). Ks: Kuolajärvi 27.VII.1934, 1 worker, 10.VII.1935, 2 workers (Krogerus).

Myrmica sulcinodis Nylander, 1846

Kk: Devichya Luda is. Lim: Apatity sur.; Kunijok r. upp.; Malyi Vudjavr 1.; Monchegorsk sur. (5 localities). Lps. Köngäs; Lotta r. (at "Sorvitsjäyr"); Pasvik Nature Reserve. 


\subsection{Family Halictidae}

Lasioglossum calceatum (Scopoli, 1763)

Ks: Kuolajärvi 7.VIII.2011 2우 (Paukkunen). Lim: Apatity 4.VIII.2014 1ठ (Kozlov); Berezovka 4.VIII.2014 10 19 (Kozlov); Kapustnoe 4.VIII.2014 2えึત (Kozlov). The record from Lps (Elfving 1968: 25) is probably based on a mislabelled female specimen collected by E. Kanervo from Lkor: Sodankylä.

Lasioglossum fratellum (Pérez, 1903)

Ks: Kutsa r. (at "ylimmäinen Jänisköngäs"); Kuolajärvi; Vuorijärvi. Lim: Berezovka; Imandra 1.; Kandalaksha; Kapustnoe; Kirovsk; Monchegorsk sur. (8 localities); Umba. Lps: Gagarka; Hakokoski; Haukilampi 1.; Kaskama; Lotta r.; Loustari; Nautsi; Nikel; Parkkina; Pitkäjärvi; Salmijärvi; Spasitelnaya Gora; Töllevi; Voryema. Lt: Pak r. low.; Verkhnetulomskiy.

Lasioglossum rufitarse (Zetterstedt, 1838)

Lim: Apatity 4.VIII.2014 1ठํ (Kozlov); province Lim (Söderman \& Leinonen 2003: 155).

Sphecodes hyalinatus Hagens, 1882

Lim: Malyi Vudjavr 1. (Fridolin 1936: 131).

\subsection{Family Megachilidae}

Megachile analis Nylander, 1852

Lv: $8 \mathrm{~km} \mathrm{~W}$. of Tetrino 2.VII.2004 1ठ̂ (Kozlov).

Osmia cf. disjuncta Tkalců, 1995

Lps: 2 km E. of Nikel 4.VII.2007 1 ㅇ (Kozlov). The specimen resembles the Mongolian species O. disjuncta, but it could also belong to an undescribed species. So far, a few similar specimens have been found from northern Finland and Sweden (Niklas Johansson, pers. comm.).

Osmia inermis (Zetterstedt, 1838)

Lt: Kola r. 7.VII.1883 2 우 (Enwald) (Sahlberg 1889: 176, as $O$. uncinata [misidentified]).

Osmia laticeps Thomson, 1872

Lps: Nautsi 1 \% (Kanervo) (Elfving 1968: 41, as O. inermis [misidentified]).

Osmia maritima Friese, 1885

Lp: 16-17 km NE. of Chapoma 30.VI.2004 1ठ 1 ㅇ (Kozlov). Lv: Kuzomen 16.-22.VI.1913
$2 \widehat{\jmath}$ (Hellén \& Frey) (Forsius \& Nordström 1921: 72). The closest known populations of this species are in southern Sweden and Norway. In both countries, the species is classified as critically endangered (Cederberg et al. 2010, Hansen et al. 2010). The distribution area extends also to the coastal areas of Denmark, Germany, the Netherlands, Poland, Mongolia, eastern Siberia, Alaska and Canada's Northwest Territories (Rightmyer et al. 2010, Müller 2011).

Osmia nigriventris (Zetterstedt, 1838)

Ks: Vuorijärvi 11.VII.1934 1ठ (Hellén). Lim: in between Kandalaksha and Zasheek 3.VII.1870 1 우 (Sahlberg) (Sahlberg 1889: 176); Malyi Vudjavr 1. (Fridolin 1936); four localities $5 \mathrm{~km} \mathrm{~N}$. to $20 \mathrm{~km} \mathrm{~S}$. of Monchegorsk 20004 exx. (Kozlov). Lps: Kaskama, $1 \hat{\jmath}$ (Grönblom).

\subsection{Family Pompilidae}

Anoplius tenuicornis (Tournier, 1889)

Lim: Kunijok r. upp. (Anufriev et al. 2011). Lps: Lotta r. (Nordström \& Forsius 1928: 3, 8, as Psammochares piliventris); Pitkäjärvi ("Kivijärvi and Laukkujoki") 20.VII.1929 1ㅇ (Storå) (Wolf 1967: 37); Voryema 13.VII.1929 1f ठ (Lindberg). $\underline{L v}$ : Kashkarantsy 20.VII.1887 $1{ }^{\lambda}$ (Levander) (Nordström \& Forsius 1928: 8, as $P$. piliventris). The record from Lps by Nordström and Forsius (1928) is probably erroneous, because it is based on specimens, which were collected by B. Poppius in August 1899, probably from the Finnish side of the Lotta river, province Li.

Arachnospila fumipennis (Zetterstedt, 1838) Lim: Kandalaksha 8.VII.1913 1ð, 1q (Hellén); Khibiny st. 4.-9.VIII.1908 1今, 1 ( Cheburova); Malyi Vudjavr r. (Fridolin 1936: 131). Lp: Pyalitsa 28.VIII.1870, 1 ( $q$ (Sahlberg) (Wolf 1967: 30). Lps: Lotta r. (Poppius) (Nordström \& Forsius 1928: 3, Wolf 1967: 30). The records from Lps by Nordström and Forsius (1928) and Wolf (1967) are based on four female specimens collected by B. Poppius in 1899 from the shores of the Lotta river. It is possible that all these specimens have been, however, collected from the province $L i$ on the Finnish side of the river. 
Arachnospila trivialis (Dahlbom, 1843)

Lim: Apatity sur. (Anufriev et al. 2011); between Kandalaksha and Zasheek 3.VII.1870 19 (Sahlberg) (Nordström \& Forsius 1928: 7, as Psammochares unguicularis); Malaya Belaya r. (Anufriev et al. 2011). Lps: Vaarlam is. (Polevoi \& Humala 2011).

Ceropales maculata (Fabricius, 1775)

Lim: Malaya Belaya r. (Anufriev et al. 2011). Lps: Lotta r. near Köngäs 14.VII.1939 1 + (Platonoff) (Wolf 1967: 43).

Dipogon bifasciatus (Geoffroy, 1785)

Lim: Malyi Vudjavr 1. (Fridolin 1936: 130).

Dipogon vechti Day, 1979

Ks: Vuorijärvi 9.VII.1934 1ठ (Hellén). Lps: Loustari 14.VII.1938 1 q (Karvonen). In Fennoscandia $D$. vechti is a rare, cavity-nesting spider wasp species, which has been red listed in Finland and Sweden (Paukkunen 2010b, Cederberg et al. 2010).

Evagetes alamannicus (Blüthgen, 1944)

Lim: Kandalaksha 30.VI.1870 1 \& (Sahlberg), 7.8.VII.1913 3 우 (Frey \& Hellén) (Nordström \& Forsius 1928: 7, as Psammochares proximus).

Evagetes sahlbergi (Morawitz, 1893)

Ks: Kuolajärvi 7.VIII.2011 1q (Paukkunen). $\underline{\text { Lps: }}$ Lotta r. near Köngäs 14.VII.1939 $1 \hat{\delta}$ (Platonoff) (Wolf 1967: 34, as E. implicatus). This species was erroneously reported from $L p s$ by Nordström and Forsius (1928: 3, 7, as Psammochares campestris) based on a female specimen collected by B. Poppius on 26.VIII. 1899 from sand dunes near Kompsiovaarat fells on the shore of the Lotta river in the province $L i$.

Priocnemis parvula Dahlbom, 1845

Lim: Malaya Belaya r. (Anufriev et al. 2011). This species was erroneously reported from Lps (Nordström \& Forsius 1928: 3-4, as P. minor, Wolf 1967: 22) on the basis of a female specimen collected by B. Poppius on 26.VIII.1899 from sand dunes near Kompsiovaarat fells on the shore of the Lotta river in the province $L i$.

\subsection{Family Sapygidae}

Sapyga similis (Fabricius, 1793)

Lim: Kukisvumchorr mt. (Fridolin 1935: 168);

Malyi Vudjavr 1. (Fridolin 1936: 130).

\subsection{Family Sphecidae}

Ammophila campestris Latreille, 1809

Lv: Kashkarantsy 25.VII.1887 19 (Levander); "Lapponia rossica" (Pulkkinen 1931: 52, Lomholdt 1976: 429). In Fennoscandia this species occurs mainly on sandy shores of the Baltic Sea. It has been classified as endangered in Finland, vulnerable in Norway and near threatened in Sweden (Paukkunen 2010b, Hansen et al. 2010, Cederberg et al. 2010). Kashkarantsy is the northernmost locality known for the species in Fennoscandia.

\subsection{Family Vespidae}

Ancistrocerus antilope (Panzer, 1798) $\underline{K s}$ : Vuorijärvi 11.VII.1934 1q (Hellén). Lt: Kola 29.VII.1913 1 + (Hellén) (Forsius \& Nordström 1923: 4).

Ancistrocerus oviventris (Wesmael, 1836)

Lim: Malyi Vudjavr 1. (Fridolin 1936: 131).

Ancistrocerus parietum (Linnaeus, 1758)

Ks: Kuolajärvi 1ㅇ (Ritavuori), 18.VI.1937 1ठ

(E. Kangas). Lim: Apatity 13.VII.2005 19 (Blinova).

Ancistrocerus scoticus (Curtis, 1826)

Lim: Kandalaksha 16.-18.VII.1870 290 (Sahlberg) (Forsius \& Nordström 1923: 7, as $A$. trimarginatus); Malyi Vudjavr 1. (Fridolin 1936: 131, as Odynerus trimarginatus). Lps: Rajakoski (Polevoi \& Humala 2011: 278); Voryema 14.VII.1930 1ठ (Frey); Zemlyanoe 1ㅇ (Kanervo). Lv (Forsius \& Nordström 1923: 7, as A. trimarginatus).

Ancistrocerus trifasciatus (Müller, 1776)

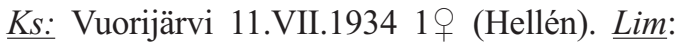
Apatity 9.VII.2005 1q (Kozlov); Malyi Vudjavr 1. (Fridolin 1936: 131); Kunijok r. upp. (Anufriev et al. 2011). Lt: Kola 6.VII.1906 1 ex. (Soldatov). 
Dolichovespula adulterina (Buysson, 1905)

Lim: Ajkuajvenjok r. 31.VII.2007 1 ex. (Blinova); Kandalaksha 25.VI.1913 19 (Hellén); Malyi Vudjavr 1. 12.VII.1934 2 exx., 9.VIII.1937 1 ex. (Fridolin). Lps: Orshoaivi 1 q (Kanervo).

Dolichovespula norwegica (Fabricius, 1781)

Lim: Chuna; Kandalaksha; Kirovsk botanical garden; Malyi Vudjavr 1.; Monchegorsk sur. (9 localities); Svetloe 1.; Umba; Umbozero 1.; Vavnbed; Vudjavrjok r.; Vudjavrchorr; Zasheek. Lm: Marjok r. low.; Porchinikha; Tumannyy sur.; Voronye; Vostochnaya Litsa. Lp: Ponoy; Tri Ostrova. L $\underline{p s:}$ : Haukilampi 1.; Kalkupää; Kiddjaur 1.; Kuvernöörinkoski; Loustari; Mattert; Menikkajoki r.; Nautsi; Parkkina; Pitkäjärvi; Trifonovo; Vayda-Guba; Vaarlam is.; Voryema; Zemlyanoe. Lt: Kola; Nota r.; Notozero 1.; Polyarnyy; Pulozero; Solovaraka; Verkhnetulomskiy. $L v$ : Kuzomen; Kuzreka; Njuemmellampjok $\mathrm{r}$.

Eumenes pedunculatus (Panzer, 1799)

Ks: Alakurtti 1.VIII.2014 1q (Kozlov).

Symmorphus allobrogus (Saussure, 1856)

$\underline{K s}$ : Kuolajärvi; Pyhäkuru; Vuorijärvi. Lim: Kandalaksha; Kanozero 1. Lps: Nautsi. Lt: Nota r.; Notozero 1. $L v$ : Olenitsa.

Symmorphus angustatus (Zetterstedt, 1838)

Lim: 16 km S. of Monchegorsk 29.VII.1991 1ठ

(Shvetsova); Malyi Vudjavr 1. (Fridolin 1936: 131, as Odynerus suecicus).

Vespula austriaca (Panzer, 1799)

Ks: Pyhäkuru. Lim: Maloe Goltsovoe 1. Lp: Chapoma sur. Lps: Longvattn; Menikkajoki r.;

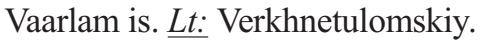

Vespula rufa (Linnaeus, 1758)

$\underline{K s}$ : Kuolajärvi; Vuorijärvi. Lim: Bolshoe Goltsovoe 1.; Kandalaksha; Malyi Vudjavr 1.; Monchegorsk sur. (3 localities). Lps: Kalkupää; Nautsi; Paz r.; Pitkäjärvi; Vaarlam is. Lt: Polyarnyy. $L v$ : Olenitsa; Varzuga.

Vespula vulgaris (Linnaeus, 1758)

Lim: Apatity; Monchegorsk sur. (2 localities). Lps: Kalkupää; Longvattn; Vaarlam is. Lt: Verkhnetulomskiy.

\section{Erroneous and doubtful records}

\subsection{Family Andrenidae}

Andrena helvola (Linnaeus, 1758)

"Lapponia rossica" (Sahlberg 1889: 173). The species has a southern distribution in Fennoscandia and therefore this record is probably erroneous.

\subsection{Family Apidae}

Anthophora furcata (Panzer, 1798)

Lim: Apatity sur. (Blinova 2010: 10, as Anthophora sp.). The specimen was reported as Anthophora sp., but if the generic affinity is correct, then apparently the species should be $A$. furcata, as no other species of the genus has been recorded in northern Fennoscandia. The closest verified records of $A$. furcata have been made south of Oulu, Finland.

Bombus norvegicus Sparre Schneider, 1918

Lim: Apatity sur.; Kirovsk botanical garden (Anufriev et al. 2011). Lim \& Lm (Söderman \& Leinonen 2003: 334). Although occurrence of this species in the Murmansk region is probable, the species is often confused with $B$. sylvestris, and therefore these records require confirmation.

Bombus rupestris (Fabricius, 1793)

Lt (Söderman \& Leinonen 2003: 327). The record from the Murmansk region on the distribution map of Söderman and Leinonen (2003) most likely represents a mistake or a typographical error, because the closest verified records are from southern Finland.

Bombus terrestris (Linnaeus, 1758)

Lim: Apatity sur. (Anufriev et al. 2011). The species has a southern distribution in Fennoscandia, and it is unlikely to find it from the Murmansk region. We suspect that the record is based on dark individuals of the Bombus lucorum group, which are easy to confuse with $B$. terrestris.

Nomada alboguttata Herrich-Schäffer, 1839

Lps: Longvattn (Polevoi \& Humala 2011: 278, Paukkunen \& Kozlov 2012: 194). The record is based on a misidentified specimen of $N$. panzeri. 


\subsection{Family Chrysididae}

Chrysis ignita (Linnaeus, 1758)

Ks: Kuolajärvi (Hellén 1920a: 213). The specimen is misidentified and represents $C$. schencki.

\subsection{Family Colletidae}

Hylaeus confusus Nylander, 1852

Lim (Söderman \& Leinonen 2003: 63). The closest verified records have been made from Tornio, Finland.

\subsection{Family Formicidae}

Formica cunicularia (Latreille, 1798)

Lim: Monchegorsk sur. (Kozlov 1997: 227-228). The record must be a mistake, as the species is restricted in Fennoscandia to southern parts of Sweden and Norway (Douwes et al. 2012).

Formica forsslundi Lohmander, 1949

Lps: Pasvik Nature Reserve and its sur. (Mershchiev 2006: 164). The record is uncertain, because the species is easy to confuse with other closely related species, and it is not known to occur any near to the Pechenga area (Douwes et al. 2012).

Formica fusca Linnaeus, 1758

Kk: Bolshaya Bogomolikha is.; Bolshoi Lomnishnyi is.; Devichya Luda is.; Dokucheikha is.; Kurichek is.; Lambin is.; Malyi Lomnishnyi is.; Ryazhkov is. (Uvarova \& Uvarov 1976: 113, Byzova et al. 1986: 281). Li: Jäniskoski (Makarova 2005: 62). Lim: Malyi Vudjavr 1. (Fridolin 1936: 134), Monchegorsk sur. (Kozlov 1997: 227-228). Lps: Pasvik Nature Reserve and its sur. (Mershchiev 2006: 164), Nikel and its sur., Pechenga (Mershchiev 2009: 127). We consider these records as uncertain, because the species has been commonly confused with $F$. lemani and $F$. gagatoides, and it has currently not been found north of the $66^{\text {th }}$ parallel in the Nordic countries (Douwes et al. 2012).

Formica pratensis Retzius, 1783

$K k$ : Devichya Luda is.; Ryazhkov is. (Uvarova \& Uvarov 1976: 114, Byzova et al. 1986: 281). Lim:
Malyi Vudjavr 1. (Fridolin 1936: 135). These records are unreliable, as the species is relatively difficult to identify and the closest verified observations are from southern Finland (Douwes et al. 2012).

Formica rufa Linnaeus, 1758

Lim: Chuna (Semenov-Tyan-Shanskiy 1975: 106); Malyi Vudjavr 1. (Fridolin 1936: 135); Monchegorsk sur. (Kozlov 1997: 227-228). The records are unreliable, as the species is often confused with other species of the Formica rufa group, and verified observations are not known from north of central Fennoscandia (Douwes et al. 2012).

Leptothorax muscorum (Nylander, 1846)

Lim (Paukkunen \& Kozlov 2012: 193). The record was based on unpublished data received from D. Dubovikoff. We consider it as uncertain, because the closest verified records of L. muscorum are from southern Finland (Douwes et al. 2012).

Monomorium pharaonis (Linnaeus, 1758)

Lim: Apatity indoors, around 2000 (A. Popova pers. comm.). This species may have been misidentified.

Myrmica rugulosa Nylander, 1849

Lps: Pasvik Nature Reserve and its sur. (Mershchiev 2006: 164). The species has a southern distribution in Fennoscandia (Douwes et al. 2012) and it is considered unlikely to occur in the Pechenga area.

\subsection{Family Halictidae}

Lasioglossum fulvicorne (Kirby, 1802)

Lim: Malyi Vudjavr 1. (Fridolin 1936: 128). Apparently, Fridolin used the name "Halictus fulvicornis" erroneously for Lasioglossum fratellum. The closest verified records of L. fulvicorne are from southern Finland.

Lasioglossum subfulvicorne (Blüthgen, 1934) Lim (Söderman \& Leinonen 2003: 152). This record is based on misidentified specimens of $L$. fratellum. 


\subsection{Family Megachilidae}

Osmia uncinata Gerstäcker, 1869

Lim: Malyi Vudjavr 1. (Fridolin 1936: 129). Lt: Kola r. (Sahlberg 1889: 176). The species is easy to confuse with other closely related species. Both specimens mentioned by Sahlberg (1889) are misidentified and belong to $O$. inermis.

\subsection{Family Vespidae}

Ancistrocerus nigricornis (Curtis, 1826)

Lim: Malyi Vudjavr 1. (Fridolin 1936: 131, as Odynerus callosus). The closest verified records of the species have been made in southern Finland (Pekkarinen \& Huldén 1991).

Dolichovespula saxonica (Fabricius, 1793)

Lim: Malyi Vudjavr 1. (Fridolin 1936: 131). The species is rare in northern Fennoscandia. It is uncertain whether Fridolin's " $V$. norvegica var. saxonica" is compatible with $D$. saxonica.

\section{Discussion}

\subsection{Species composition}

A total of 123 species of aculeate Hymenoptera are recorded from the Murmansk region. The records of an additional 16 species are considered as uncertain, and the records of three species are erroneous. The majority of the aculeates found in the region has wide distribution areas and are more common in southern Fennoscandia. Several of these species have their northernmost known localities in the Murmansk region. These include the ant Formica polyctena, the digger wasps Ammophila campestris, Crossocerus varus and Lindenius albilabris and the bees Megachile analis, Osmia cf. disjuncta and Bombus veteranus. At least $B$. veteranus has probably only recently dispersed to the Kola Peninsula, because it has not been recorded earlier from the area despite its easy detectability.

About ten species found in the Murmansk region are confined to mountainous and/or northern regions in Europe. The bumblebees Bombus alpinus, B. hyperboreus, B. monticola and B. po- laris, which live in the treeless tundra of northern and eastern Kola Peninsula, and also in the Khibiny mountains, are particularly well adapted to cold conditions. Other northern species include the bumblebees B. balteatus and B. lapponicus, the digger wasps Crabro maeklini and Gorytes neglectus and the dryinid wasp Anteon subarcticum. Several species, such as $B$. cingulatus, B. flavidus, B. sporadicus and Formica gagatoides, have a wider northern boreal distribution in Fennoscandia.

\subsection{Level of knowledge of the fauna}

Although the total number of species, confirmed by the examined material, is relatively high for such a northern location, many more species are still expected to be found from the Murmansk region. This suggestion is supported by the high proportion of species that have been collected from the region only once ( 33 of 123 species, i.e. $27 \%$ ). According to the checklist of Söderman and Vikberg (2003), a total of 230 aculeate species have been found from the approximately same sized area of northern Finland which includes the following Finnish biogeographical provinces: Ostrobottnia borealis pars borealis $(O b b)$, Regio kuusamoënsis $(K s)$, Lapponia kemensis pars occidentalis (Lkoc), Lapponia kemensis pars orientalis (Lkor), Lapponia enontekiensis $(\mathrm{Le})$ and Lapponia inarensis $(\mathrm{Li})$. Currently, the species number for northern Finland is even somewhat larger as several new species have been found there, and some species have been split into two or more species during the past decade. Therefore we estimate that about 100 additional species could still be found from the Murmansk region. Knowledge of the distribution and abundance of different species remains also at a relatively low level. This is exemplified by the fact that Bombus sporadicus was included as the only hymenopteran in the Red Book of the Murmansk region (Shutova 2003), although it is common and widespread in the area.

Knowledge of the aculeate species composition varies considerably in different parts of the Murmansk region (Fig. 2). Of the biogeographical provinces, the best studied are Lapponia imandrae $(\mathrm{Lim})$, from which a total of 86 species 
Fig. 2. Collecting sites of aculeate Hymenoptera in the Murmansk region. Number of recorded species is shown by black dots of different size: small dot: 1-5 species, mediumsized dot: 6-10 species, large dot: 11-44 species. Locality names, coordinates and species numbers are presented in Table 1.

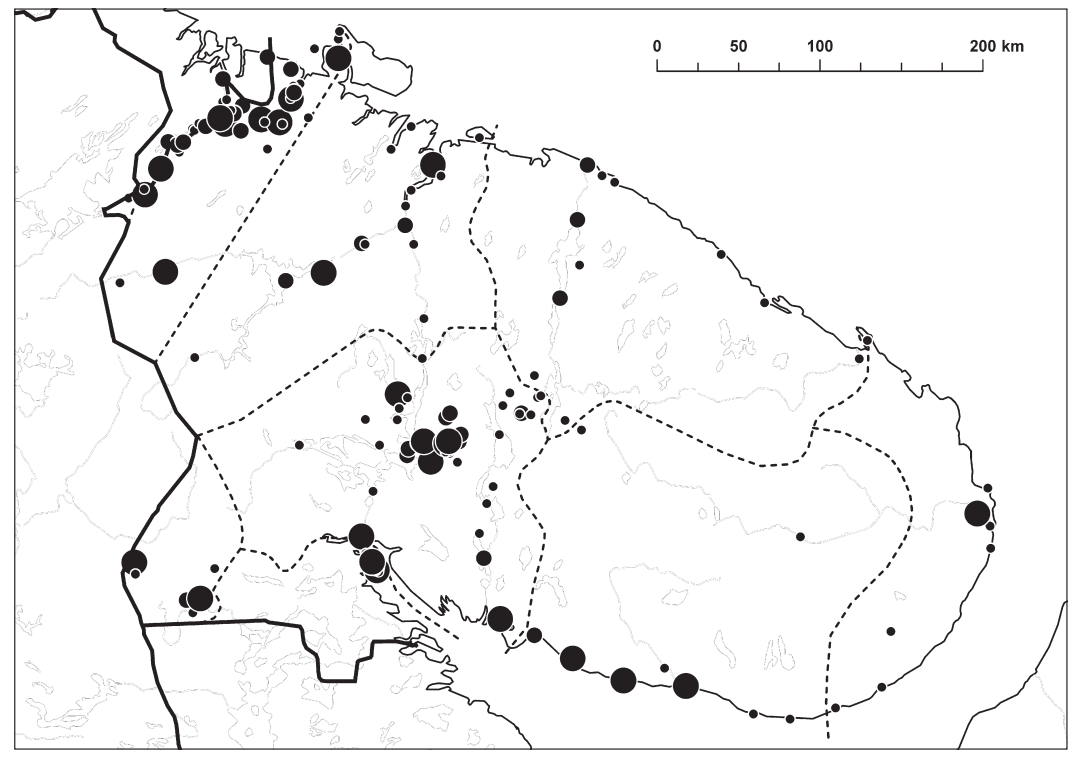

have been found. The province Lapponia petsamoënsis (Lps) is also fairly well studied, with a total of 65 species. Clearly less well known are Lapponia murmanica $(L m), 14$ species, and Lapponia ponojensis $(L p), 20$ species, due to their remote locations. Also the northern part of Karelia keretina $(K k)$ is still poorly studied, with only 18 recorded species. The aculeates of the Russian part of Regio kuusamoënsis $(K s)$ with 55 species, Lapponia varsugae $(L v)$ with 39 species, and Lapponia tulomensis $(L t)$ with 33 species, also remain relatively poorly studied. In the future, more efforts should be made to survey hymenopterans and other insects in these fascinating, but poorly known areas of northern Europe.

Acknowledgements. Dmitry A. Dubovikoff, Pekka Punttila, Jouni Sorvari, Guy Söderman and Veli Vikberg took part in the determination of the aculeate material. Ilona Blinova allowed us to use her unpublished manuscript of the pollinators of orchids in the Murmansk region. Kirsi Valanne helped us in preparing the maps, an anonymous reviewer made valuable improvements to the text, and Stephen Venn checked the English language.

\section{References}

Aguiar, A. P., Deans, A. R., Engel, M. S., Forshage, M., Huber, J. T., Jennings, J. T., Johnson, N. F., Lelej, A. S., Longino, J. T., Lohrmann, V., Mikó, I., Ohl, M.,
Rasmussen, C., Taeger, A. \& Yu, D. S. K. 2013: Order Hymenoptera. - In: Zhang, Z.-Q. (ed.), Animal biodiversity: An outline of higher-level classification and survey of taxonomic richness (Addenda 2013). - Zootaxa 3703: 51-62.

Anufriev, G. A., Efremova, Z. A., Mokrousov, M. V. \& Zryanin, V. A. 2011: (List of Hymenoptera species found from Khibiny and Lovozero mountains) [www document in Russian]. URL http:/www.biodiversity.ru/kola/html/khibiny/pril_14.html. (Site visited on 31 December, 2011)

Bertsch, A. 2009: Barcoding cryptic bumblebee taxa: $B$. lucorum, B. crytarum and B. magnus, a case study (Hymenoptera: Apidae: Bombus). — Beiträge zur Entomologie 59: 287-310.

Blinova, I. V. 2010: (To the knowledge of insect species composition in the Murmansk region.) - In: Evdokimova, G. A. \& Vandysh, O. I. (eds.), (Biodiversity conservation issues in northern regions): 9-10. PolarAlpine Botanical Garden-Institute, Apatity-Kirovsk. 51 pp. [In Russian.]

Blinova, I. V. \& Mironov, V. G. 2012: New records of spiders and insects for NE Fennoscandia (Murmansk Region, Russia). - Memoranda Societatis pro Fauna et Flora Fennica 88: 80.

Byzova, Yu. B., Uvarov, A. V., Gubina, V. G., Zalesskaja, N. T., Zaharov, A. A., Petrova, A. D., Suvorov, A. A. \& Vorobjeva, E. G. 1986: (Soil invertebrates of the White Sea islands in the Kandalaksha Nature Reserve.) - Nauka, Moscow. 312 pp. [In Russian.]

Cederberg, B., Abenius, J., Hellqvist, S., Högmo, O., Larsson, M. \& Sörensson, M. (2010) Steklar - Wasps, Hymenoptera. - In: Gärdenfors, U. (ed.), Rödlistade arter i Sverige 2010 - The 2010 Red List of Swedish Species: 345-360. ArtDatabanken, SLU, Uppsala. 590 pp. 
Cederberg, B. \& Nilsson, L. A. 2010: Osmia maritima. Havsmurarbi [www document]. URL http://www.artfakta.se/Artfaktablad/Osmia_Maritima_102686.pdf. (Site visited on 31 December, 2011)

Collingwood, C. A. 1979: The Formicidae (Hymenoptera) of Fennoscandia and Denmark. — Fauna Entomologica Scandinavica 8: 1-174.

Douwes, P., Abenius, J., Cederberg, B., Wahlstedt, U., Hall, K., Starkenberg, M., Reisborg, C. \& Östman, T. 2012: Nationalnyckeln till Sveriges flora och fauna. Steklar: Myror-getingar. Hymenoptera: FormicidaeVespidae. - ArtDatabanken, SLU, Uppsala. 382 pp.

Elfving, R. 1968: Die Bienen Finnlands — Fauna Fennica 21: $1-69$.

Enwald 1881: (Meeting report: Bombus hyperboreus found from Russian Lapland.) - Meddelanden af Societas pro Fauna et Flora Fennica 6: 255. [In Swedish.]

Forsius, R. \& Nordström, A. 1921: Verzeichnis der aus Finland bisher bekannten Apiden. — Notulae Entomologicae 1: 70-77.

Forsius, R. \& Nordström, Å. 1923: Zur Kenntnis der Vespiden Finnlands. — Notulae Entomologicae 3: 1-9.

Frey, R. 1915: En resa till Kola-halfön. — Terra 27: 111126.

Friese, H. 1911: Neue Varietäten von Bombus (Hym.) III. — Deutsche entomologische Zeitschrift 1911: 571572.

Fridolin, V. Yu. 1935: Koukiss-woum-tschorr, le plateau central des Monts Khibines au point de vue biocénotique. - Entomologicheskoe Obozrenie 26: 165-182.

Fridolin,V. Yu. 1936: (Animal and plant community of the Khibiny mountains. Biocenotic research in 19301935.) - Proceedings of the Kola branch of the Academy of Sciences of the USSR, vol. 3, Acad. Sci. publ. house, Moscow \& Leningrad. 295 pp. [In Russian.]

Gjershaug, J. O., Staverløkk, A., Kleven, O. \& Ødegaard, F. 2013: Species status of Bombus monticola Smith (Hymenoptera: Apidae) supported by DNA barcoding. - Zootaxa 3716: 431-440.

Grönblom, T. 1916: (Meeting report: Three insect species new to Finland.) - Meddelanden af Societas pro Fauna et Flora Fennica 42: 28-31. [In Swedish.]

Hansen, L. O., Lønnve O. J. \& Ødegaard, F. 2010: Veps. Hymenoptera. - In: Kålås, J. A., Viken, Å., Henriksen, S. \& Skjelseth, S. (eds.) 2010, The 2010 Norwegian Red List for Species: 331-346. Norwegian Biodiversity Information Centre, Norway. 480 pp.

Hellén, W. 1920a: Über finländische Goldwespen. Meddelanden af Societas pro Fauna et Flora Fennica 46: 203-213.

Hellén, W. 1920b: Zur Kenntnis der Bethyliden und Dryiniden Finlands. - Meddelanden af Societas pro Fauna et Flora Fennica 45: 277-289.

Hellén, W. 1953: Übersicht über die Bethyliden und Dryiniden Finnlands. - Notulae Entomologicae 33: 88102.

Hellén, W. 1954: Sphecidenfunde aus Finnland (Hym.). Notulae Entomologicae 34: 121-130.

Hellén, W. 1955: Sphecidenfunde aus Finnland II (Hym.). — Notulae Entomologicae 35: 65-68.
Kozlov, M. V. 1997: Pollution impact on insect biodiversity in boreal forests: evaluation of effects and perspectives of recovery. - In: Crawford, R. M. M. (ed.), Disturbance and Recovery in Arctic Lands: An Ecological Perspective: 213-250. Proceedings of the NATO Advanced Research Workshop on Disturbance and Recovery of Arctic Terrestrial Ecosystems, Rovaniemi, Finland, 24-30 September 1995. (NATO ASI series. Partnership subseries 2. Environment: vol. 25). Kluwer Academic Publishers, Dordrecht. $621 \mathrm{pp}$.

Kozlov, M. V. \& Jalava, J. 1994: Lepidoptera of Kola Peninsula, Northwestern Russia. - Entomologica Fennica 5: 65-85.

Kozlov, M. V. \& Kullberg, J. 2011 [2010]: New and interesting records of Lepidoptera from the Kola Peninsula, Northwestern Russia, in 2000-2009. — Entomologica Fennica 21: 254-272.

Kozlov, M. V., Zvereva, E. L. \& Zverev, V. E. 2009: Impacts of point polluters on terrestrial biota. - Springer, Dordrecht. 466 pp.

Lomholdt, O. 1975: The Sphecidae (Hymenoptera) of Fennoscandia and Denmark, part 1. - Fauna Entomologica Scandinavica 4: 1-224.

Lomholdt, O. 1976: The Sphecidae (Hymenoptera) of Fennoscandia and Denmark, part 2. - Fauna Entomologica Scandinavica 4: 225-452.

Løken, A. 1973: Studies on Scandinavian Bumble Bees (Hymenoptera, Apidae). - Norwegian Journal of Entomology 20: 1-218.

Makarova, O. A. (ed.) 2005: Annals of Nature of Pasvik State Nature Reserve 9 (2002). — Ryazan State Pedagogical University, Ryazan. 148 pp. [In Russian.]

Mershchiev, A. V. 2006: (Northern taiga ant fauna of the Pasvik Nature Reserve and its surroundings.) - In: Evdokimova, G. A. \& Vandysh, O. I. (eds.), (Environmental problems of the north. Proceedings of the international conference, 10-12 October 2006, part 2): 163-165. Kola Science Centre, Apatity. 246 pp. [In Russian.]

Mershchiev, A. V. 2009: (Myrmecological monitoring of anthropogenically disturbed areas in the forest tundra of the Kola Peninsula.) - Vestnik of Moscow State Regional University, Ser. Natural Sciences No 3: 125 130. [In Russian.]

Müller, A. 2011: Palaearctic Osmiine Bees. ETH Zürich [www document]. URL http://blogs.ethz.ch/osmiini. (Site visited on 31 December, 2011)

Nordström, Å. \& Forsius, R. 1928: Über die Psammocharidae (Pompilidae) Finlands. — Notulae Entomologicae 8 : 1-13.

Olmi, M. 1994: The Dryinidae and Embolemidae (Hymenoptera: Chrysidoidea) of Fennoscandia and Denmark. - Fauna Entomologica Scandinavica 30: 1100.

Paukkunen, J. 2010a: Checklist of aculeate Hymenoptera of Finland [www document]. URL http://taxon.luomus.fi. (Site visited on 31 December, 2013)

Paukkunen, J. 2010b: Stinging wasps. — In: Rassi, P., Hyvärinen, E., Juslén, A. \& Mannerkoski, I. (eds.), The 2010 Red List of Finnish Species: 529-544. Ympäris- 
töministeriö \& Suomen ympäristökeskus, Helsinki. $685 \mathrm{pp}$.

Paukkunen, J. \& Kozlov, M. V. 2012: Kuolan niemimaan myrkkypistiäiset. - In: Vanhatalo, A., Niemelä, P., Kuuluvainen, T. \& Vasander, H. (eds.), Turjan vuonoilta Vienanmerelle: matka Kuolan niemimaan luontoon ja kulttuuriin: 189-200. Helsingin yliopiston metsätieteiden laitoksen julkaisuja 4. Helsingin yliopiston metsätieteiden laitos, Helsinki. 220 pp.

Paukkunen, P., Rosa, P., Soon, V., Johansson, N. \& Ødegaard, F. 2014: Faunistic review of the cuckoo wasps of Fennoscandia, Denmark and the Baltic countries (Hymenoptera: Chrysididae). - Zootaxa 3864: 1-67.

Pekkarinen, A. \& Huldén, L. 1991: Distribution and phenology of the Ancistrocerus and Symmorphus species in eastern Fennoscandia (Hymenoptera, Eumenidae). — Entomologica Fennica 2: 179-189.

Pekkarinen, A. \& Huldén, L. 1995: Distribution and phenology of the Vespinae and Polistinae species in eastern Fennoscandia (Hymenoptera: Vespidae). — Sahlbergia 2: 99-111.

Pekkarinen, A., Teräs, I., Viramo, J. \& Paatela, J. 1981: Distribution of bumblebees (Hymenoptera, Apidae: Bombus and Psithyrus) in eastern Fennoscandia. Notulae Entomologicae 61: 71-89.

Pilgrim, E., von Dohlen, C., \& Pitts, J. 2008: Molecular phylogenetics of Vespoidea indicate paraphyly of the superfamily and novel relationships of its component families and subfamilies. - Zoologica Scripta 37: 539-560.

Polevoi, A. V. \& Humala, A. E. 2011: (Insects.) - In: Polikarpova, N. V. (ed.), (Annals of the Pasvik Nature Reserve. Book 15 (2008)): 153-156, 264-281. Kola Science Centre, Apatity. 314 pp. [In Russian.]

Pulawski, W. 2011: Catalog of Sphecidae (= Apoidea excluding Apidae) [www document]. URL http://research.calacademy.org/ent/catalog_sphecidae. (Site visited on 31 December, 2011)

Pulkkinen, A. 1931: Suomen eläimet, Animalia Fennica 1, Myrkkypistiäiset, Hymenoptera Aculeata 1, Petopistiäiset, Sphecidae. — WSOY, Porvoo. 168 pp.

Rightmyer, M. G., Griswold T., \& Arduser M. S. 2010: A review of the non-metallic Osmia (Melanosmia) found in North America, with additional notes on palearctic Melanosmia (Hymenoptera, Megachilidae). ZooKeys 60: 37-77.

Rikkinen, K. 1980: Suuri Kuolan retki 1887. — Otava, Helsinki. 189 pp.

Sahlberg, J. 1889: Catalogus praecursorius Hymenopterorum Anthophilorum Fennicae. - Meddelanden af Societas pro Fauna et Flora Fennica 15: 167-178.

Sahlberg, J. 1910a: Om parasitstekel-släktet Gonatopus och dess Finska representanter (Hym.). - Acta Societas pro Fauna et Flora Fennica 33(7): 1-19.

Sahlberg, J. 1910b: Lyhyt katsaus Suomen kulta-ampiaisiin (Chrysidae). — Luonnon Ystävä 14: 94-99.

Semenov-Tyan-Shanskiy, O. I. 1975: (Lapland Nature Reserve.) - Murmansk publ. house, Murmansk. 244 pp. [In Russian.]

Sharkey, M. 2007: Phylogeny and classification of Hymenoptera. - Zootaxa 1668: 521-548.

Shutova, E. V. 2003: Bombus sporadicus Nylander, 1848. - In: Konstantinova, N. A., Koryakin, A. S. \& Makarova, O. A. (eds.), (Red book of the Murmansk region): 260-261. Murmansk publ. house, Murmansk. 400 pp. [In Russian.]

Silfverberg, H. 1988: Finnish Entomologists on the Kola Peninsula. - Notulae Entomologicae 68: 115-120.

Skorikov, 1912: (Bombus lapponicus (F.) and its varieties (Hymenoptera, Bombidae).) - Russian Review of Entomology 12: 95-102. [In Russian.]

Skorikov, A. S. 1914: (Hortobombus consobrinus (Dahlb.) and its varieties (Hymenoptera, Bombidae).) - Russian Review of Entomology 14: 283-286. [In Russian.]

Söderman, G. \& Leinonen, R. 2003: Suomen mesipistiäiset ja niiden uhanalaisuus. - Tremex Press Oy, Helsinki. 420 pp.

Söderman, G. \& Vikberg, V. 2003 [2002]: Suomen myrkkypistiäisten luettelo ja levinneisyys. - Sahlbergia 7: 41-66.

Soon, V., Budrys, E., Orlovskyte, S., Paukkunen, J., Ødegaard, F., Ljubomirov, T. \& Saarma, U. 2014: Testing the validity of Northern European species in the Chrysis ignita species group (Hymenoptera: Chrysididae) with DNA barcoding. - Zootaxa 3786: 301-330.

Striganova, B. R. 1973: (Soil fauna of the northern coast of the Kola Peninsula.) - In: Gilyarov, M. S. (ed.), (Ecology of soil invertebrates): 75-84. Nauka, Moscow. 226 pp. [In Russian.]

Urbanavichus, G., Ahti, T. \& Urbanavichene, I. 2008: Catalogue of lichens and allied fungi of Murmansk region, Russia. - Norrlinia 17: 1-80.

Uvarova, L. A. \& Uvarov, A. V. 1976: (Materials to the study of the ant fauna of the northern archipelago of Kandalaksha bay.) - In: Gilyarov, M. S. (ed.), (Fauna and ecology of invertebrates): 112-114. Moscow State Pedagogical Institute, Moscow. 158 pp. [In Russian.]

Wolf, H. 1967: Wegwespen (Hym. Pompiloidea) Finnlands. - Acta Entomologica Fennica 23: 1-46.

Zvereva, E. L., Kruglova, O. Y. \& Kozlov, M. V. 2010: Drivers of host plant shifts in the leaf beetle Chrysomela lapponica: natural enemies or competition? - Ecological Entomology 35: 611-622. 